\title{
Orion Entry Display Feeder and Interactions with the Entry Monitor System
}

\author{
Darren Baird ${ }^{1}$, Mike Bernatovich ${ }^{6}$, Ellen Gillespie ${ }^{6}$, Binaifer Kadwa $^{6}$, Dave Matthews ${ }^{2}$, \\ Wes Penny ${ }^{3}$, Tim $Z^{2}{ }^{8}$ \\ NASA Johnson Space Center, Houston, TX, 77058
}

\begin{abstract}
The Orion spacecraft is designed to return astronauts to a landing within $10 \mathrm{~km}$ of the intended landing target from low Earth orbit, lunar direct-entry, and lunar skip-entry trajectories. While the landing is nominally controlled autonomously, the crew can fly precision entries manually in the event of an anomaly. The onboard entry displays will be used by the crew to monitor and manually fly the entry, descent, and landing, while the Entry Monitor System (EMS) will be used to monitor the health and status of the onboard guidance and the trajectory. The entry displays are driven by the entry display feeder, part of the Entry Monitor System (EMS). The entry re-targeting module, also part of the EMS, provides all the data required to generate the capability footprint of the vehicle at any point in the trajectory, which is shown on the Primary Flight Display (PFD). It also provides caution and warning data and recommends the safest possible re-designated landing site when the nominal landing site is no longer within the capability of the vehicle. The PFD and the EMS allow the crew to manually fly an entry trajectory profile from entry interface until parachute deploy having the flexibility to manually steer the vehicle to a selected landing site that best satisfies the priorities of the crew. The entry display feeder provides data from the EMS and other components of the GNC flight software to the displays at the proper rate and in the proper units. It also performs calculations that are specific to the entry displays and which are not made in any other component of the flight software. In some instances, it performs calculations identical to those performed by the onboard primary guidance algorithm to protect against a guidance system failure. These functions and the interactions between the entry display feeder and the other components of the EMS are described.
\end{abstract}

Attitude Indicators

The PFD is shown in Figure 1. The PFD can be broken up into 3 main areas. The first area, shown in the blue outline, consists of spacecraft attitude indicators, attitude errors, bank angle queues. The Attitude Director Indicator (ADI) ball provides a 3D representation of the spacecraft attitude in a Local Vertical Local Horizontal (LVLH) reference frame. These attitudes are provided as Euler angles, which are converted within the entry display feeder, from a direction cosine matrix using a pitch, yaw, roll Euler sequence. The corresponding attitude rates and errors are shown on the graduated bar scale on the top, right, and bottom of the ADI ball. During entry, the crew can manually control the bank angle but not the pitch and yaw angles. Pitch and yaw forces are dominated by aerodynamics effects and cannot be significantly affected by thrusters during entry, so those channels are disabled. The current bank angle is displayed with the magenta airplane symbol, while the commanded bank angle is displayed with the green airplane symbol. The commanded bank angle is smoothed with a low-pass filter with a time constant of 0.2 seconds to prevent jumpiness that would distract a crew. The vertical, magenta needle shows the bank angle error, and the entry display feeder calculates the direction of the

${ }^{1}$ GNC Engineer, JSC-DM42

${ }^{2}$ Aerospace Engineer, JSC-C0B

${ }^{3}$ Ascent/Entry Guidance and Procedures Instructor, JSC-DM43 
needle consistent with the shortest time and least fuel consumption while operating independently of any guidance and control algorithms. To fly a precision entry the crew must have a means to anticipate the time to the next bank reversal, which is shown along with a digital readout of the current delta-azimuth on the digital display below the ADI ball. This estimate is based on the current lateral angle, current lateral angle rate, and lateral angle deadband. A bank reversal is triggered when the lateral angle exceeds the lateral angle deadband and is consistent with the primary entry guidance algorithm, PredGuid. These attitude indicators provide the crew with the visual queues to modulate bank in a manner consistent with PredGuid while flying manually. As currently designed, the backup guidance algorithm, Loads Managed Guidance (LMG), does not execute bank reversals, so this display is disabled when LMG is active.

\section{Entry Footprint Displav}

The second area of the PFD consists of the entry footprint and bank angle displays, shown in the red outline on Figure 1. The current lift direction, consistent with the current bank angle, is shown with an arrow on the dial display. The entry footprint display provides the crew with geographic situational awareness during entry. Because the crew is flying backwards through the atmosphere, the footprint is oriented upside down (South is on the top and North is on the bottom) such that the California coastline is shown on the bottom of the display. This display orientation provides the crew with consistency between the bank commands and the predicted landing site. Throughout entry, the entry re-targeting module calculates all of the onboard trajectory propagations and passes the data to the footprint display via the entry display feeder. The target landing site is indicated by the circle labeled "SNC" (San Clemente Island), and the predicted landing site assuming a current, constant bank angle is indicated by the parachute. The predicted landing site of the backup guidance algorithm, Loads-Managed Guidance (LMG) is indicated by the "L," and the ballistic landing site is indicated by the " $\mathrm{B}$." The capability footprint is indicated by the closed polygon, which shows the predicted landing sites at constant bank angles of $0^{\circ}$ (full lift up), $\pm 30^{\circ}, \pm 60^{\circ}, \pm 90^{\circ}$, and $180^{\circ}$ (full lift down). To the right of the footprint are the bank angles, and the corresponding maximum G-loads that would be encountered when flying these constant-bank trajectories to parachute deploy. When the target landing site falls outside of the capability footprint due to a spacecraft fault or trajectory error, the onboard retargeting module of the EMS recommends a new landing site to the crew via an alert in the yellow area of the footprint display. It also recommends the safest reachable re-targeted landing site based on a pre-loaded list of contingency areas. If none of those sites are available, the re-targeted landing site is recommended based on a pre-loaded relative factor of safety matrix that underlies the entry footprint display. The footprint display provides the crew with the visual queues to manually fly to the safest possible landing site within the capability of the vehicle.

\section{Additional Flight Control Indicators}

The third area of the PFD consists of basic flight control indicators, excluding attitude indicators. These data are passed directly from the absolute navigation domain of the GNC flight software to the displays via the entry display feeder and are shown in the orange outlines on Figure 1. The dial displays show altitude, altitude rate, and current acceleration (G-load). The yellow and red G-load limits of $10 \mathrm{gs}$ and 12gs, respectively, are also on the acceleration indicator. Earth-relative velocity is shown on a tape display to the left of the ADI ball. These basic flight control indicators complement the other displays on the PFD 
and provide the pilots with additional situational awareness necessary to fly an entry manually.

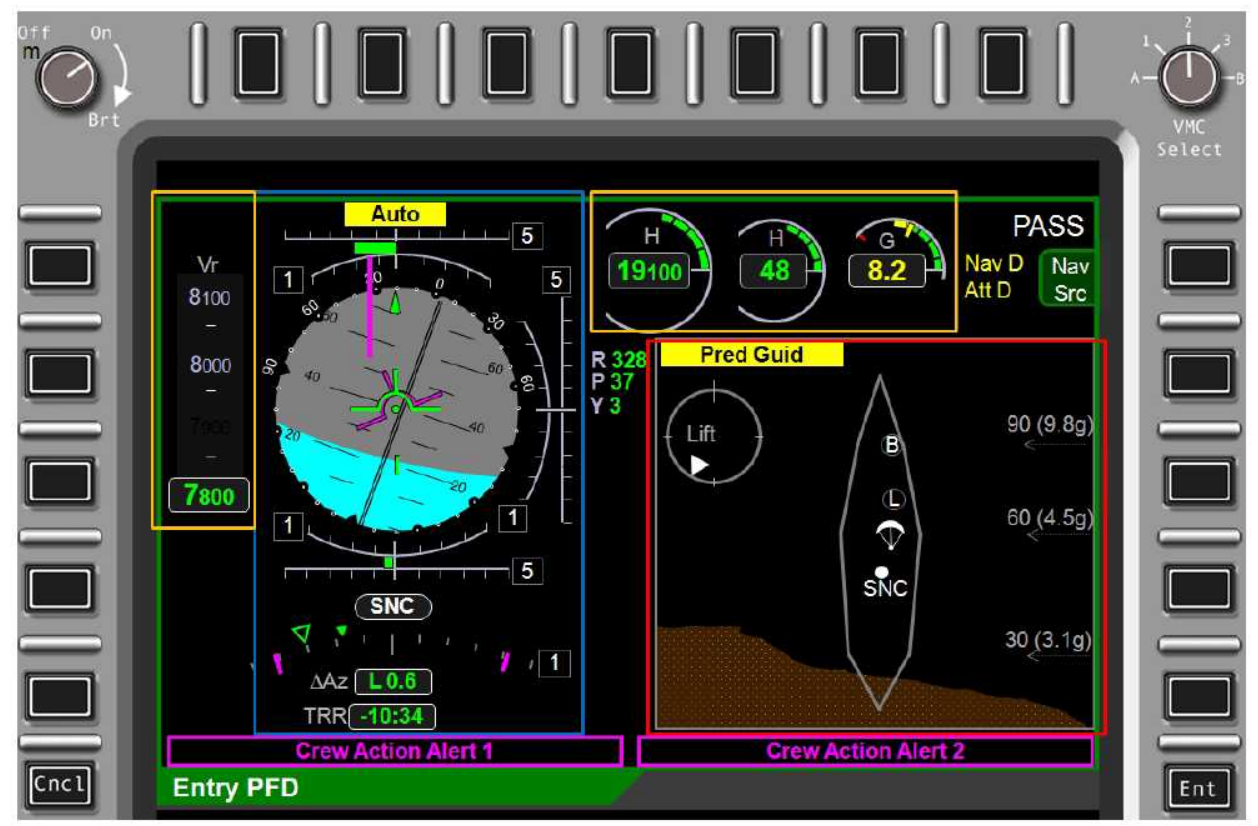

Figure 1: Entry Primary Flight Display 


\section{Introduction}

\section{Orion Project}

- Orion Entry Monitor System (EMS) monitors the health and status of the onboard guidance and the trajectory

- Navigation monitor provides health and status information regarding the navigation solution

- Fuel monitor estimate the amount of fuel required to successfully complete the entry, descent, and landing sequence

- If insufficient fuel remains, fuel-saving options will be recommended, including downmoding to another guidance algorithm, eliminating bank reversals, or enlarging the guidance over-control gain.

- The guidance monitor provides health and status of the guidance algorithms

- If anomaly is detected, recommendations will be made to downmode from the Predictor Guidance (PredGuid) algorithm to the Precision Loads-Managed Guidance (PLMG) algorithm or a ballistic entry

- Entry re-targeting module computes the current trajectory and the capability footprint of the vehicle and determines if the selected landing site is within the capability of the vehicle

- If the selected landing site falls outside of the capability of the vehicle, a new landing site is recommended to the crew

- The entry display feeder is the conduit between the GNC flight software and the onboard displays

- Operates at $10 \mathrm{~Hz}$

- Operates under all guidance modes, including manual control 

System

Orion Project

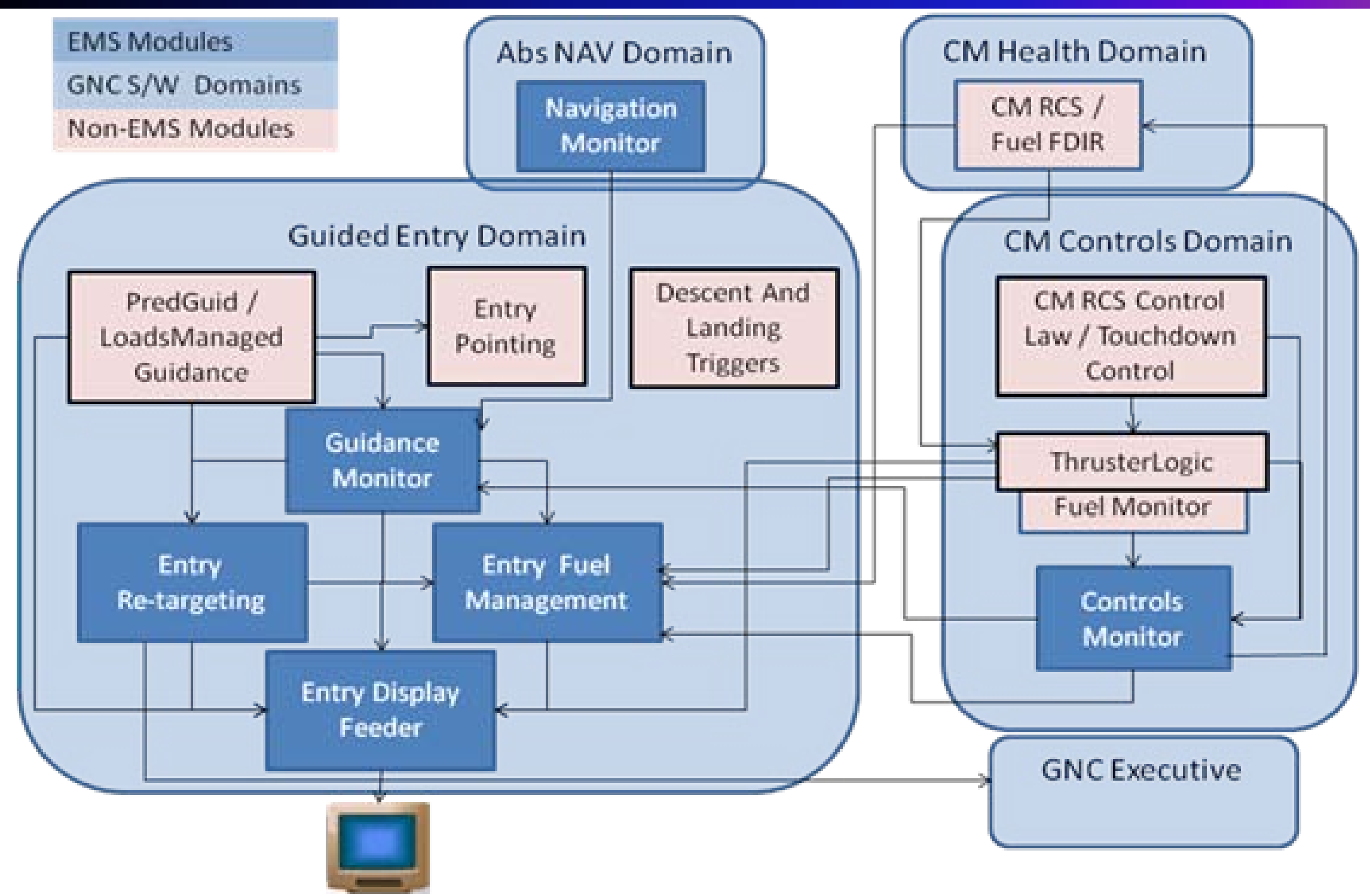




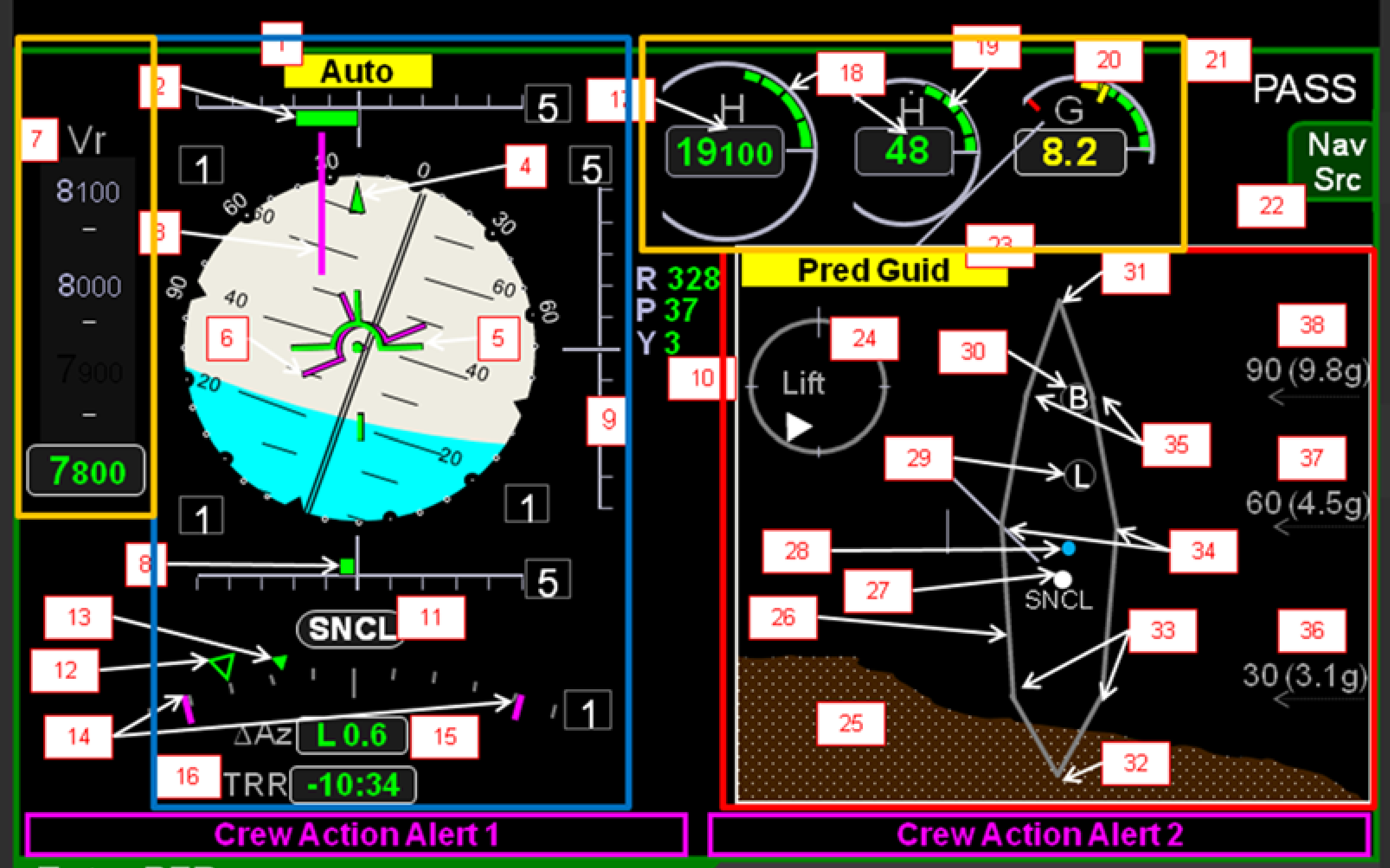

\section{Entry PFD}




\section{Bank Error Smoothing}

\section{Orion Project}

- Bank error computed within the entry display feeder as difference between the actual and commanded bank angles

- Vertical, magenta bank error needle provides precise bank error information to crew

- Entry display feeder provides an option to smooth the bank error needle signal with a low-pass filter with a time constant of $2 \mathrm{~s}$ to prevent jumpiness that would distract a crew

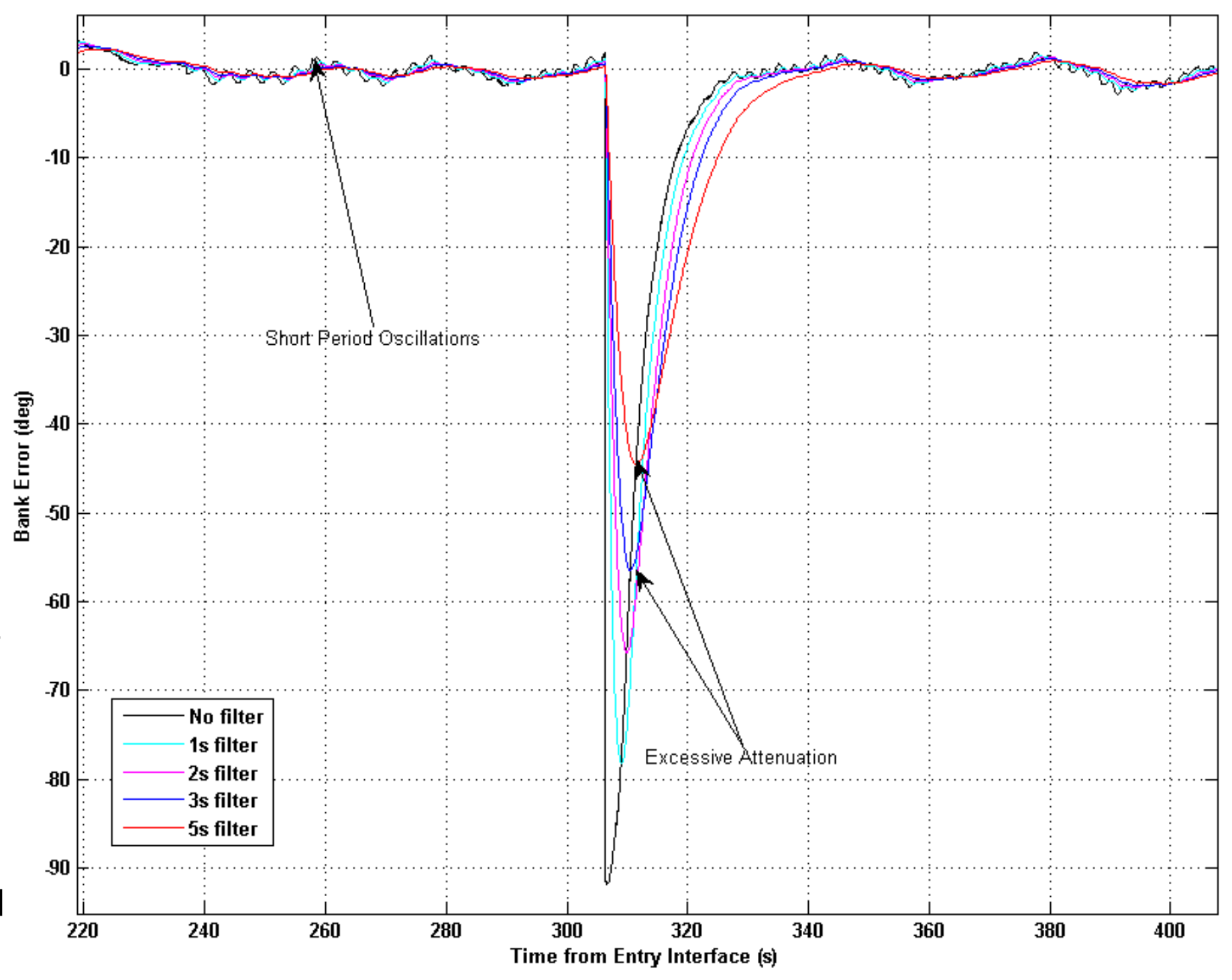


- The entry display feeder calculates the direction to bank consistent with the shortest time and least fuel consumption

- Operates independently of any guidance and control algorithms

- Useful during manual control

- Not necessarily the direction of shortest angular distance

- Minimum time to bank left or bank right calculated (includes phase wrapping)

- Switching function (u) chosen based on shortest time

$u= \begin{cases}+1 & x-\frac{\dot{x}|x|}{2 a}>0 \\ -1 & x-\frac{\dot{x}|x|}{2 a}<0\end{cases}$

$\mathrm{x}=$ bank error

$\mathrm{a}=$ constant roll acceleration

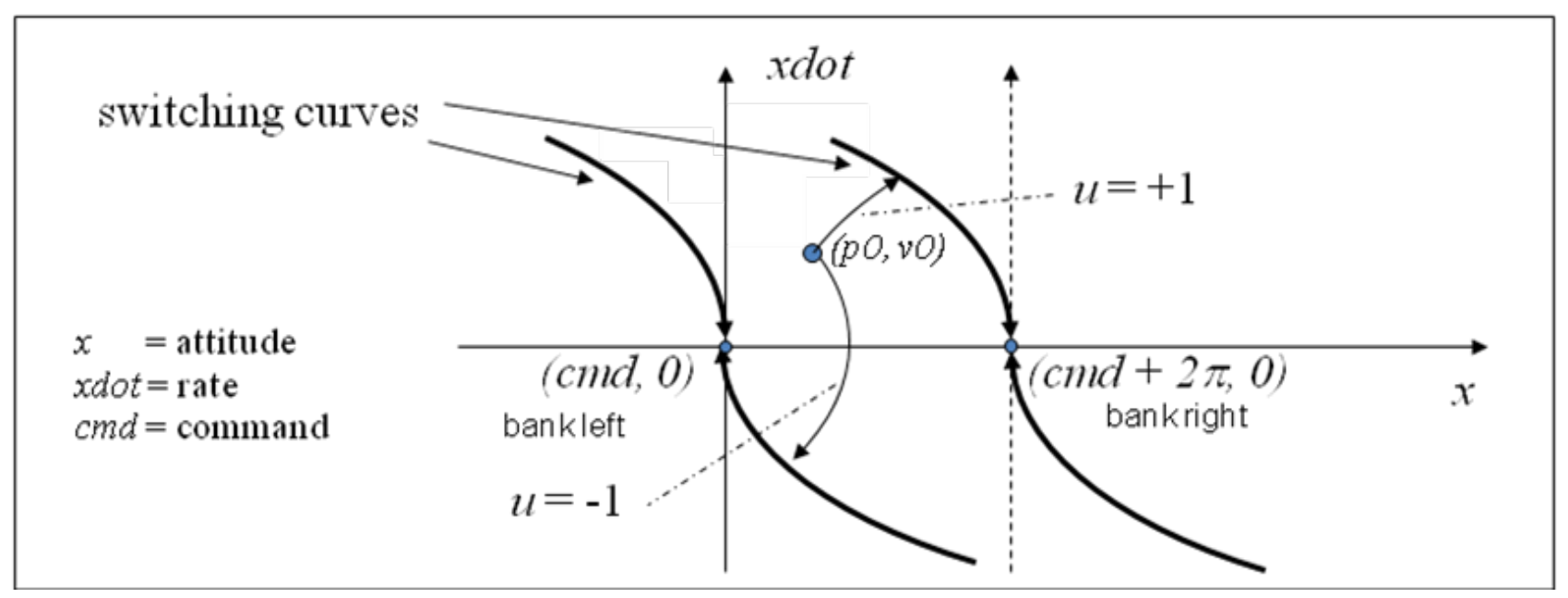


- Peak bank rate is found when the time to bank is resolved

- If peak rate exceeds vehicle rate limit, the time to bank is adjusted with a rate- limited time to bank.

- Solutions for the left and right bank directions are compared and the appropriate bank error is selected.

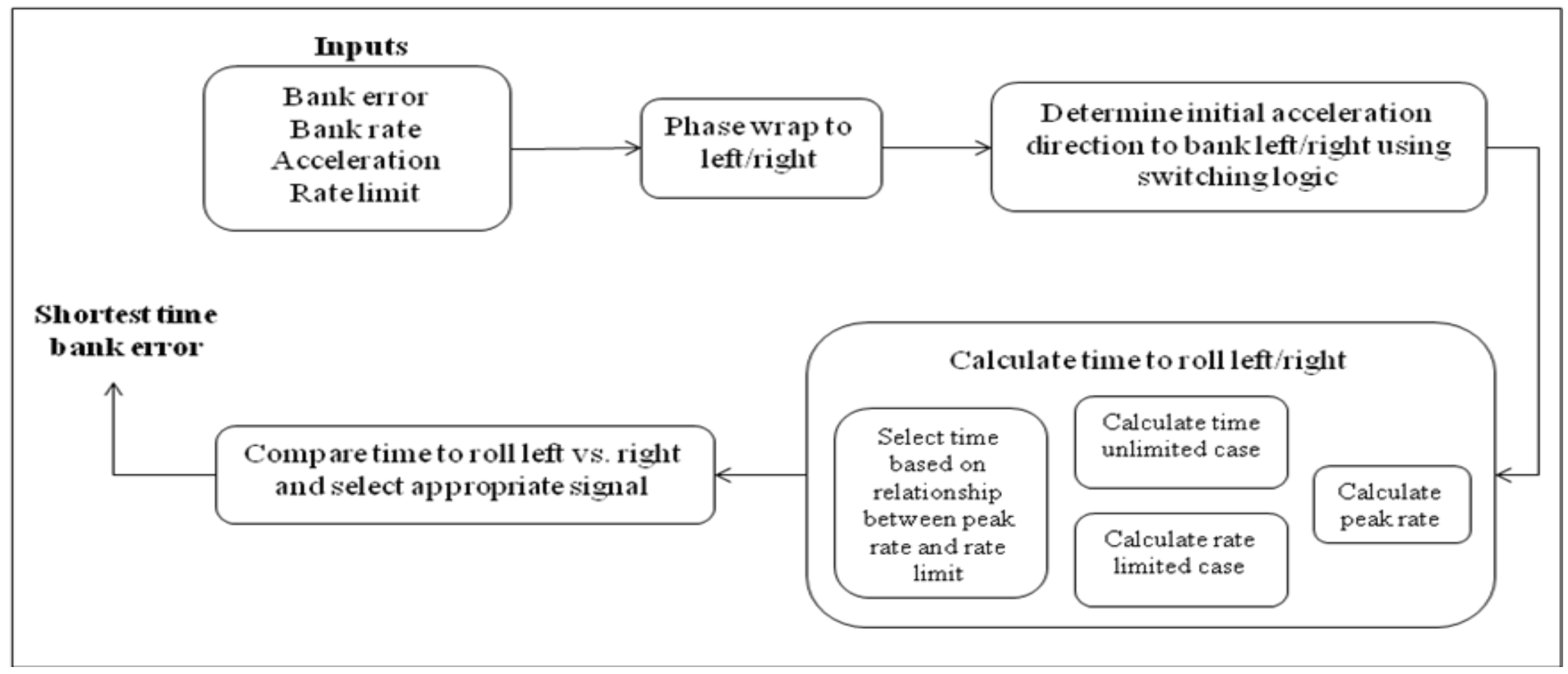




\section{Example of Shortest Time Logic}
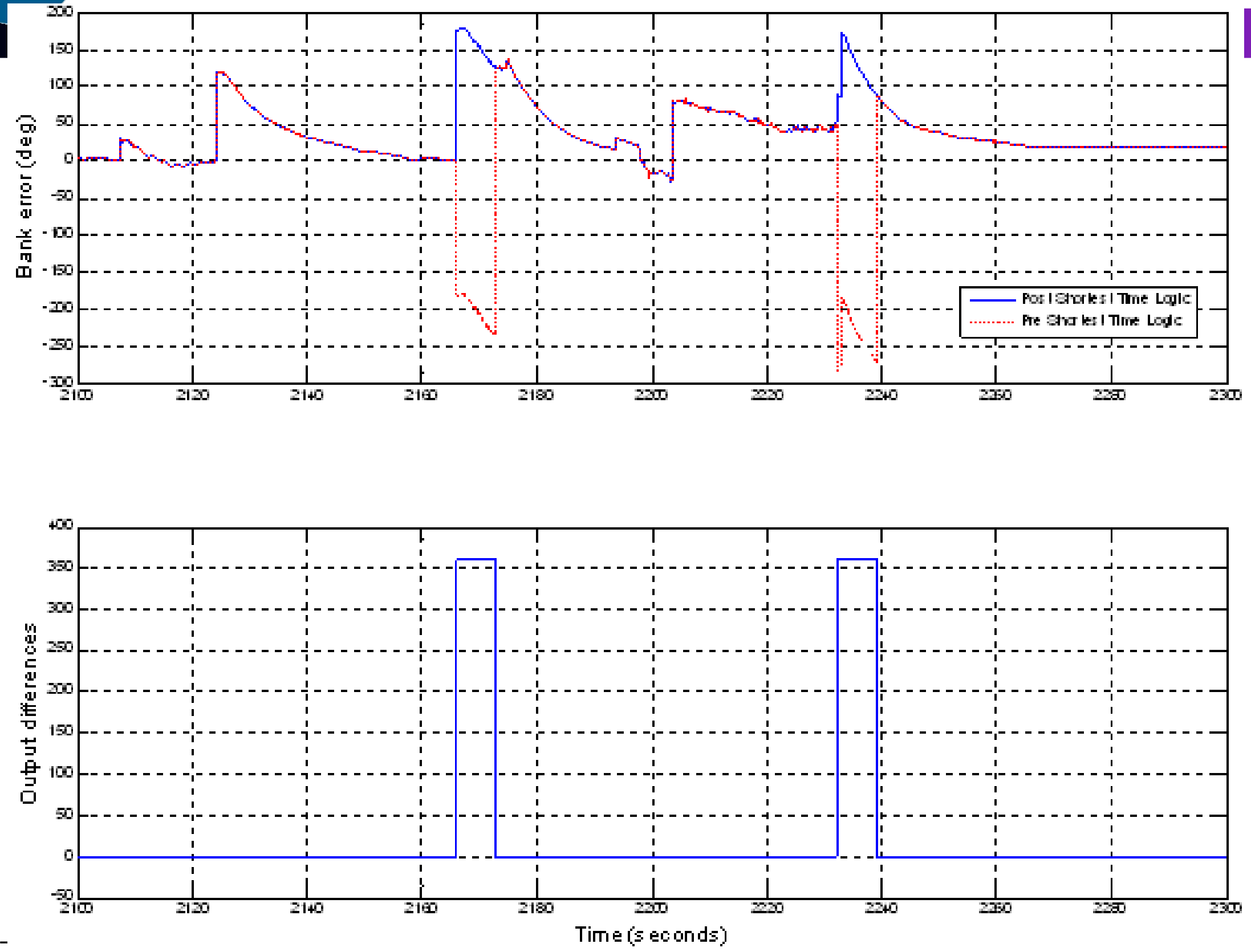
- Heading error is computed as the angle between the trajectory plane, determined by the vehicle position and velocity vectors, and the target plane, determined by the vehicle and target landing site position vectors.

- When on the primary guidance algorithm, a bank reversal is triggered when the lateral angle exceeds the lateral corridor limits

- The current lateral angle and lateral angle 15

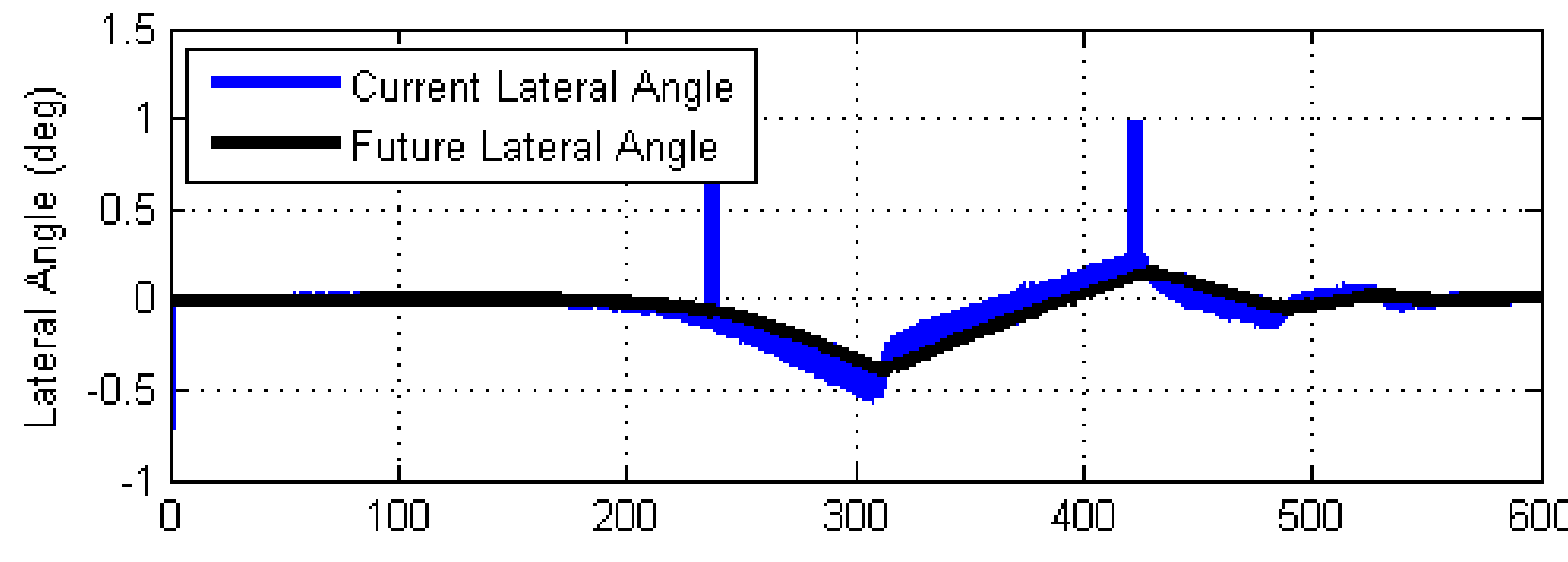
seconds in the future are shown with respect to the lateral angle limits in the bottom left of the PFD

- Errors as large as 0.2 near time of bank reversal

- Time to bank reversal estimate more appropriate in these regions

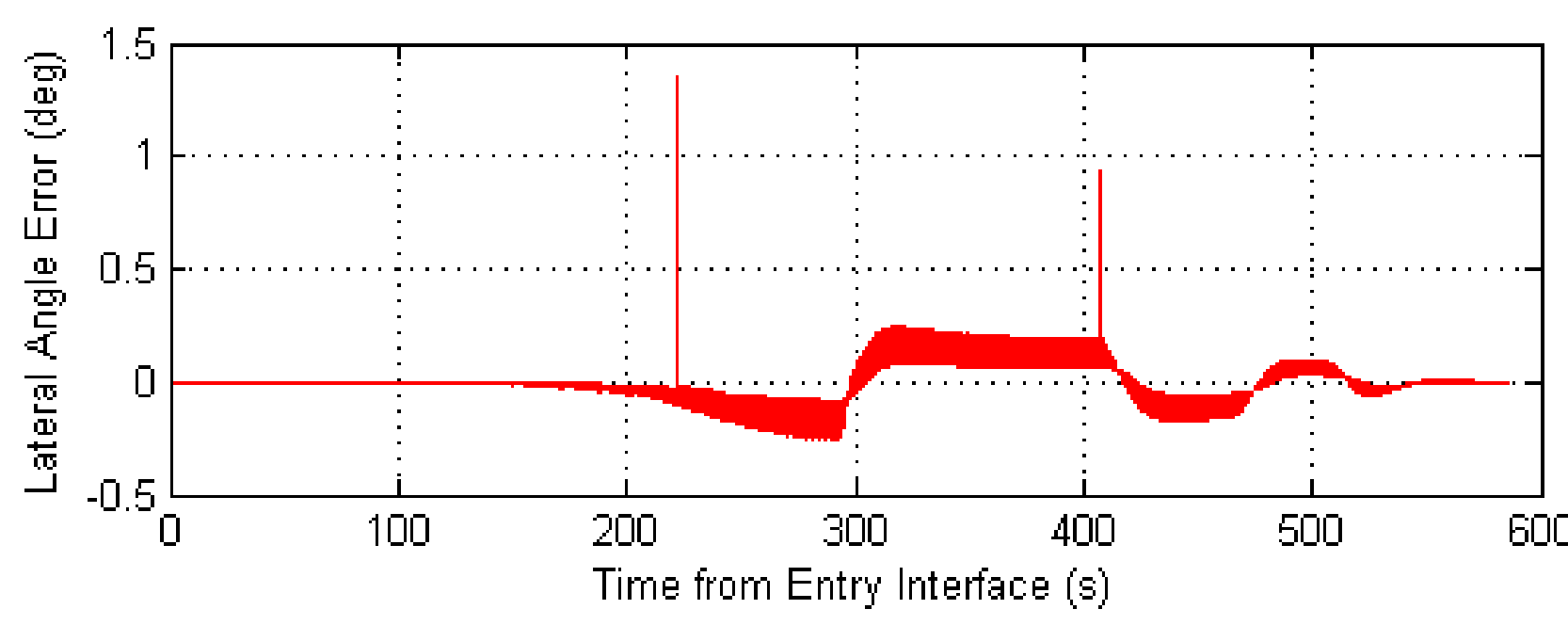


- Current lateral angle is propagated using the current lateral angle rate

- Time to reach the current lateral corridor is calculated using current constant lateral angle rate.

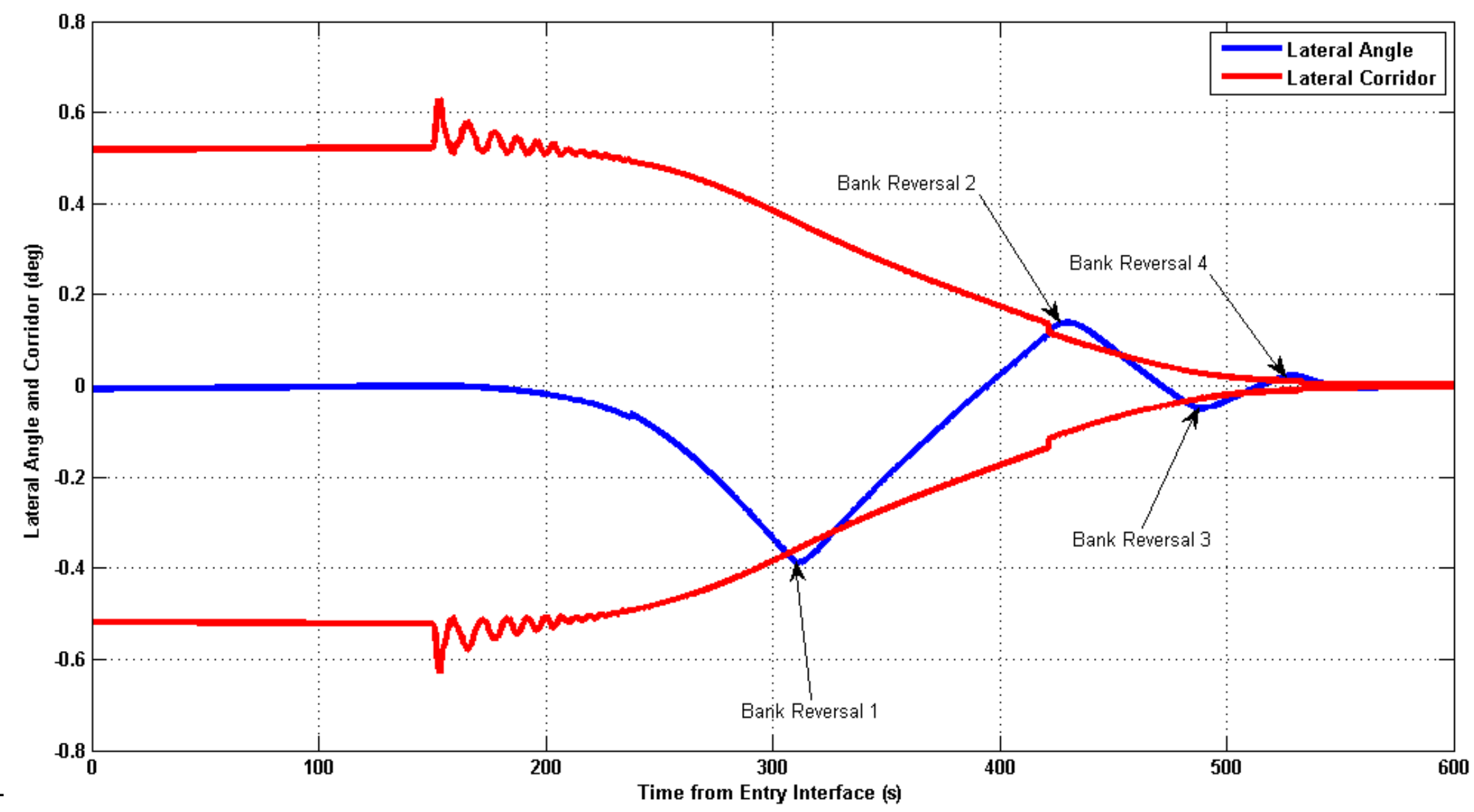




\section{NASA PredGuid Time to Bank Reversal Prediction}

\section{Orion Project}

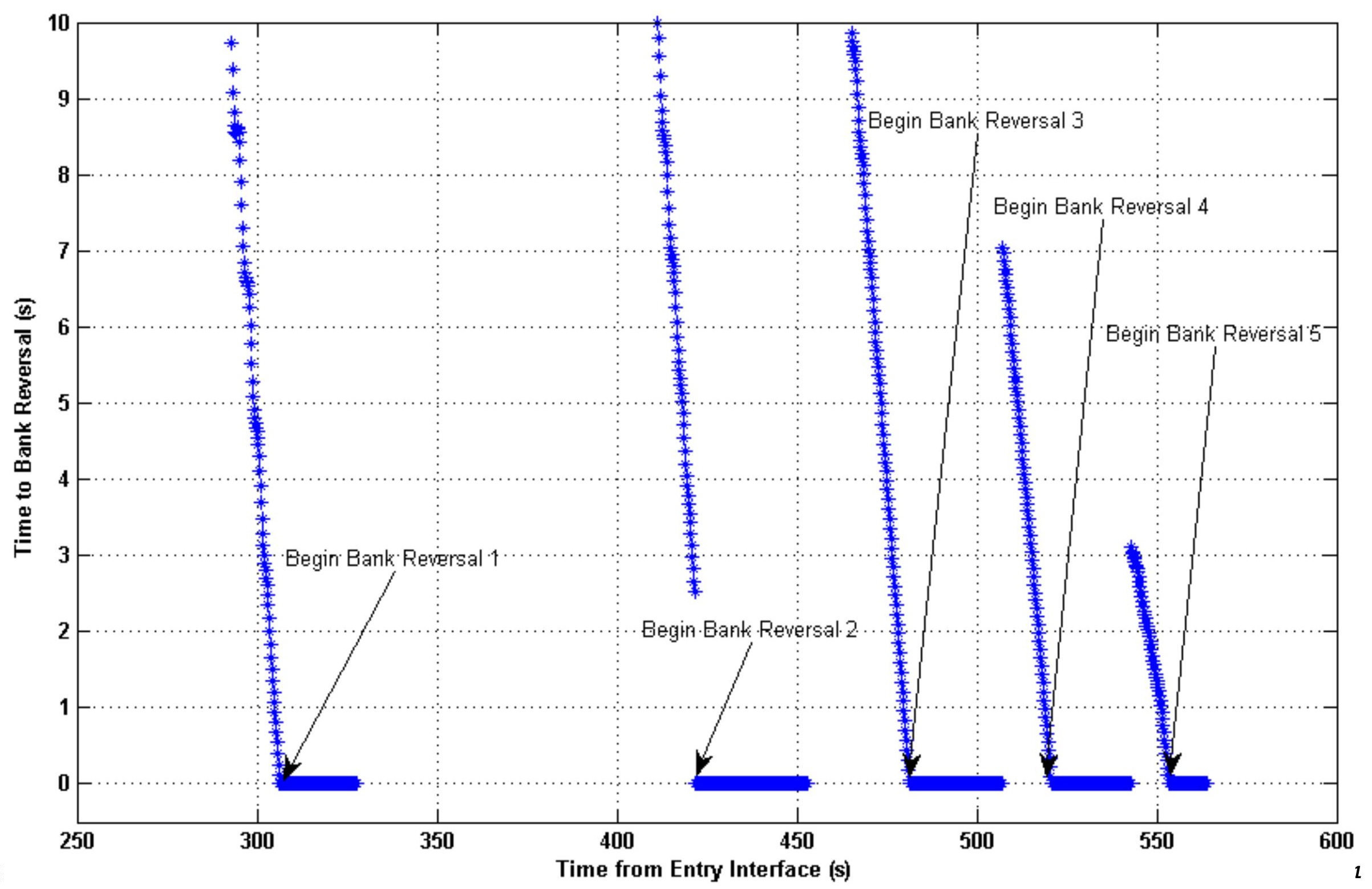



Errors

Orion Project

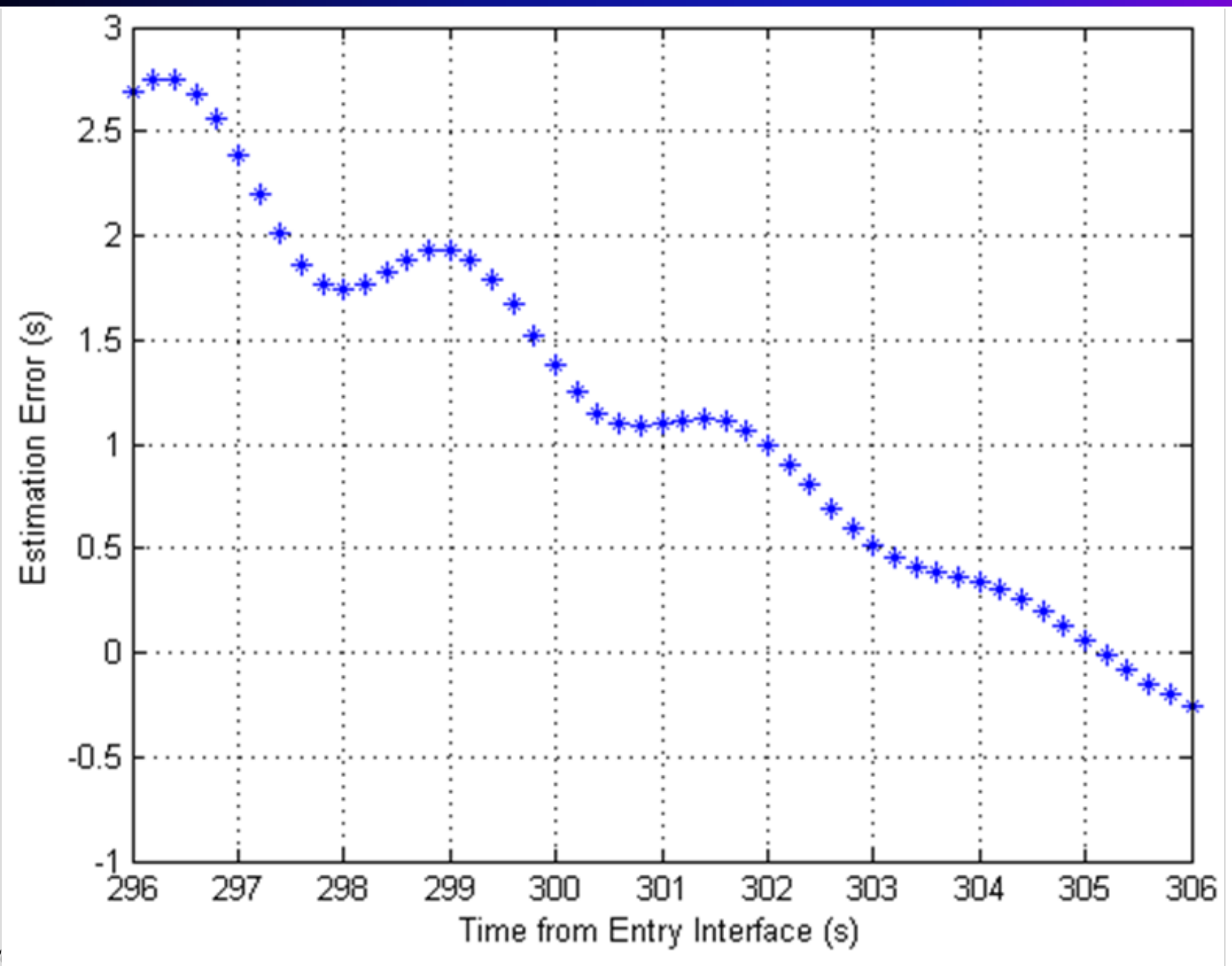




\section{Time to Bank Reversal Prediction on Loads- Managed Guidance}

\section{Orion Project}

Time_To_Bank_Reversal_ $P L M G=\left(\right.$ Gload $\left.^{*} p v 2\right) /\left(V_{\text {rel_LMG }}-V_{\text {Bank }}\right)$

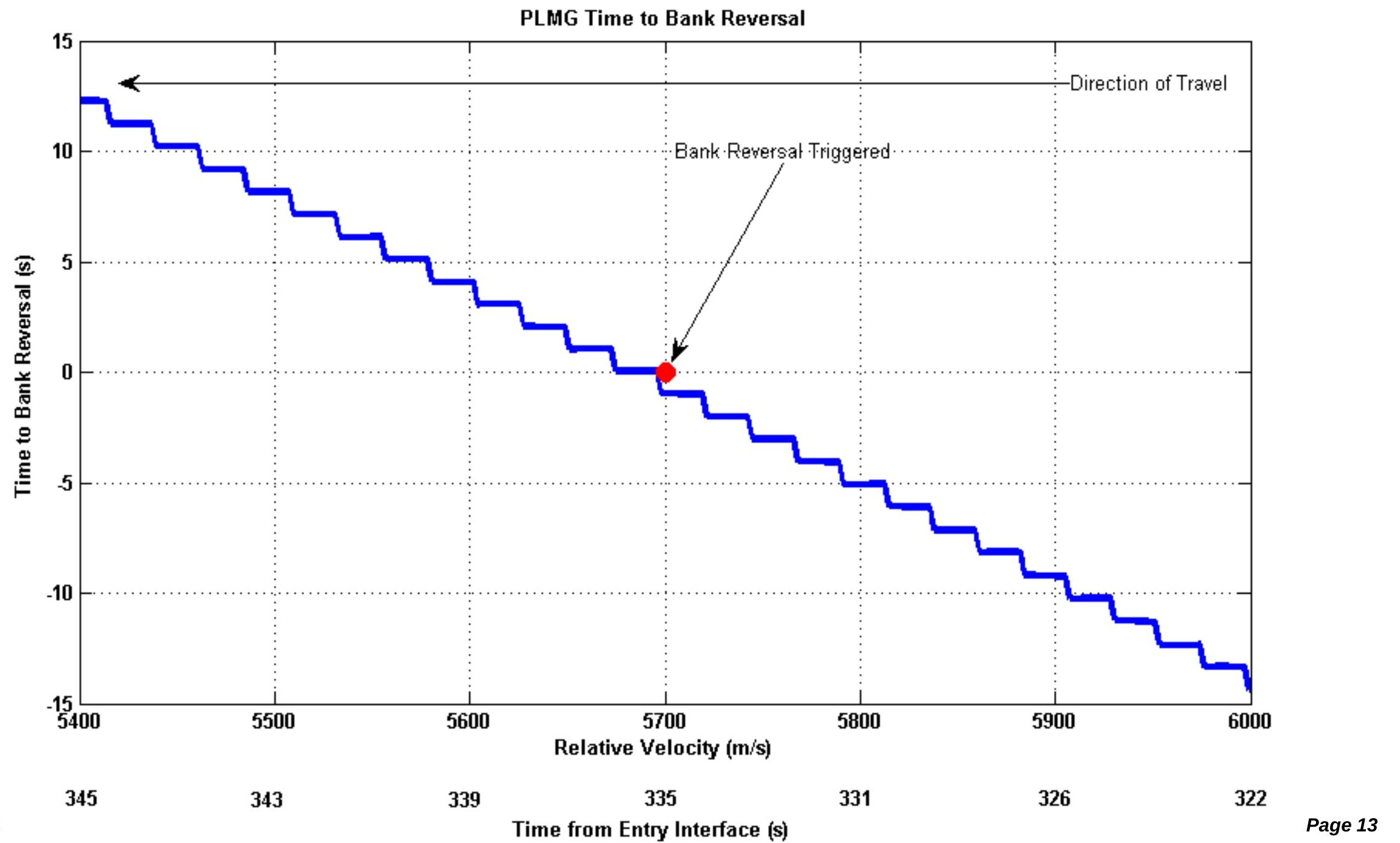




\section{Entry Footprint Display}

Orion Project

- Provides crew with geographic situational awareness during entry

- Entry re-targeting module propagates all constant-bank trajectories

- Used to define footprint on display, which is passed through the display feeder

- Used to recommend new landing site if target falls outside of capability footprint

- G-loads for constant bank angle trajectories shown to right of footprint
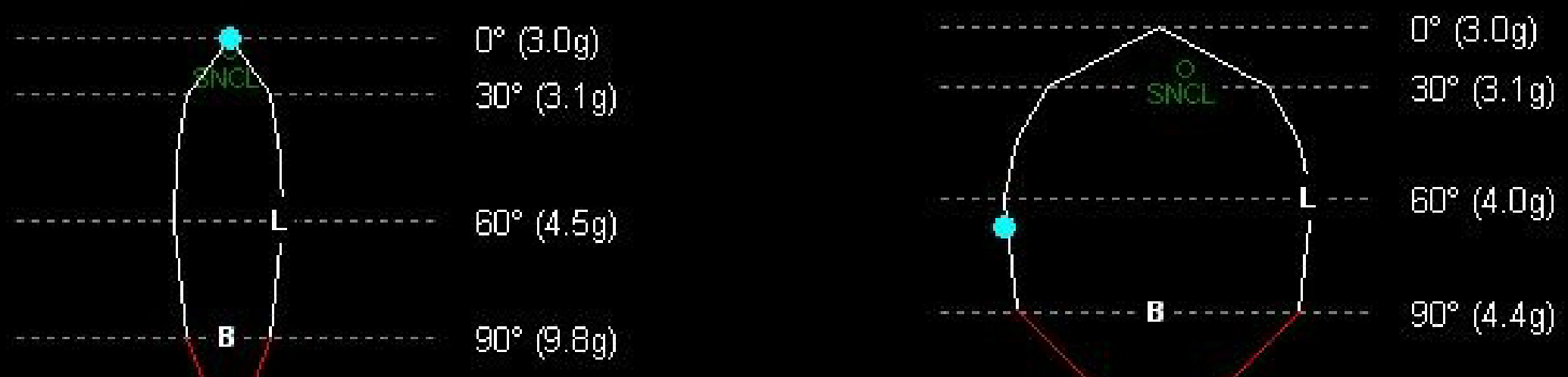
- Indicated by orange outlines on slide 4

- Dial displays

- Altitude (ft)

- Altitude rate (ft/s)

- Altitude acceleration (ft/s $\left.{ }^{2}\right)$

- Calculated within entry display feeder

- Calculated by differentiating altitude rate

- Current acceleration (Gs)

- Tape display

- Earth-relative velocity (ft/s) 


\section{Additional Entry Display Feeder Work}

- Feed entry trajectory displays

- Smoothing of lateral corridor to remove oscillations in time to bank reversal estimations

- Increase performance of entry re-targeting module for entry footprint generation

- Calculate180\%10G landing point and display on footprint 


\section{Conclusions}

- Entry display feeder is an integral part of the Orion entry monitor system

- Serves as conduit between multiple GNC flight software domains and displays

- Responsible for outputting data in proper units

- Generates display data not provided by other GNC flight software

- Smoothing of bank error data

- Shortest time logic in the bank error signal for use in manual control

- Prediction of time to next bank reversal (for both PredGuid and Loads-Managed Guidance)

- Next major step is adding capability to drive entry trajectory displays 
- Matthews, D., "Ascent Abort Flight Display Suite, Pass 1 Description," NASA Johnson Space Center, 2009.

- Bilimoria, K., email, 11 September 2009, karl.bilimoria@nasa.gov.

- Bryson, A E, Jr. and Ho, Y C. Applied Optimal Control: Optimization, Estimation, and Control, Hemisphere, 1975, pp. 111-113.

- Loe, G., e-mail, 16 March 2010 and 26 March 2010, Greg.Loe@honeywell.com.

- Yazell, D., e-mail, 04 May 2010 and 26 March 2010, Douglas.Yazell@honeywell.com.

- Putnam, Z.R., Neave, M.D., and Barton, G.H., "PredGuid Entry Guidance for Orion Return from Low Earth Orbit," IEEE paper \#1571, 7 January 2009.

- Johnson, W., Personal Interview, 5 April 2010. 


\title{
Orion Entry Display Feeder and Interactions with the Entry Monitor System
}

\author{
Darren Baird ${ }^{1}$, Mike Grant ${ }^{2}$, and Bini Kadwa ${ }^{3}$, \\ NASA Johnson Space Center, Houston, TX, 77058, USA \\ Ellen Gillespie ${ }^{4}$, Dave Matthews ${ }^{5}$, Wes Penny ${ }^{6}$, and Tim Zak ${ }^{7}$ \\ United Space Alliance, Houston, TX, 77058, USA \\ and \\ Brian Bihari ${ }^{8}$ \\ Hamilton Sundstrand, Webster, TX 77598, USA
}

\begin{abstract}
The Orion spacecraft will be capable of returning astronauts to a landing within $10 \mathrm{~km}$ of the intended landing target from low Earth orbit. The onboard entry displays will be used by the crew to monitor entry, descent, and landing. The entry displays are driven by the entry display feeder, part of the Entry Monitor System (EMS). The entry re-targeting module, also part of the EMS, provides all the data required to generate the capability footprint of the vehicle at any point in the trajectory, which is shown on the Primary Flight Display (PFD). It also recommends the safest possible re-designated landing site when the nominal landing is no longer within the capability of the vehicle, and it calculates the energy bucket, which is shown on the entry trajectory displays. These displays will allow the crew to fly like entry guidance flies throughout the entry trajectory while having the flexibility to manually steer the vehicle to best satisfy the priorities of the crew. The entry display feeder provides data from the EMS and other components of the GNC flight software to the displays at the proper rate and in the proper units. It also performs calculations that are specific to the entry displays and which are not made in any other module of the flight software. These functions and the interactions between the entry display feeder and the other components of the EMS are described, and snapshots of salient data on the entry displays are discussed.
\end{abstract}

\section{Nomenclature}

$C M \quad=$ Crew Module

EI $\quad=$ Entry Interface

EMS = Entry Monitor System

FDIR = Fault Detection, Isolation, and Recovery System

GNC = Guidance, Navigation, and Control

PFD = Primary Flight Display

$P L M G \quad=$ Precision Loads-Managed Guidance

${ }^{1}$ GNC Engineer, Ascent/Descent Flight Design Division, 2101 NASA Pkwy, MC DM42, AIAA Senior Member.

${ }^{2}$ GNC Engineer, Ascent/Descent Flight Design Division, 2101 NASA Pkwy, MC DM42, AIAA Member.

${ }^{3}$ GNC Engineer, Ascent/Descent Flight Design Division, 2101 NASA Pkwy, MC DM42, AIAA Member.

${ }^{4}$ GNC Engineer, Ascent/Descent Flight Design Division, 2101 NASA Pkwy, MC DM42, AIAA Member.

${ }^{5}$ Aerospace Engineer, Astronaut Office, 2101 NASA Pkwy, MC CB, AIAA Member.

6 Ascent/Entry Guidance and Procedures Instructor, Ascent \& Descent Flight Dynamics Division, 2101 NASA Pkwy, MC DM43, AIAA Member.

7 Ascent/Entry Guidance and Procedures Instructor, Ascent \& Descent Flight Dynamics Division, 2101 NASA Pkwy, MC DM43, AIAA Member.

${ }^{8}$ CEV Entry GN\&C Project Lead, Engineering Science Contract Group, 455 E. Medical Center Blvd. 


\section{Introduction}

$\mathrm{T}$

$\neg$ HE Orion spacecraft is designed to return astronauts to a landing within $10 \mathrm{~km}$ of the intended landing target from low Earth orbit. The Entry Monitor System (EMS) will be used to monitor the health and status of the onboard guidance and the trajectory. A diagram of the preliminary design of the EMS is shown in Figure 1. Orion GN\&C flight software is broken out by domains, and EMS modules are contained in the absolute navigation, Crew Module (CM) health management, CM controls, and the guided entry domains. In the absolute navigation domain resides the navigation monitor, which provides health and status information regarding the navigation solution. In the event that the navigation solution is suspect or not useful, the navigation monitor will pass this information to the guidance monitor. The CM health management domain contains and CM Reaction Control System (RCS) and fuel Fault Detection, Isolation, and Recovery (FDIR, pronounced fi-der) monitor. In the event of a propulsion system hardware malfunction such as a stuck thruster, inoperative thruster, or fuel leak, FDIR will send information to the entry fuel management monitor directly and via the fuel monitor, located within the CM controls domain, to better estimate the amount of fuel required to successfully complete the entry, descent, and landing sequence. The guided entry domain contains most of the entry monitor software. The guidance monitor will monitor the health of the guidance algorithms and make recommendations to downmode from the Predictor Guidance (PredGuid) algorithm to the Precision Loads-Managed Guidance (PLMG) algorithm or a ballistic entry in the event that an anomaly is detected. The fuel management monitor will evaluate current fuel usage with respect to the mission timeline and estimate the fuel required to complete the entry, descent, and landing sequence. If insufficient fuel remains, fuelsaving options will be recommended, including downmoding to another guidance algorithm, eliminating bank reversals, or enlarging the guidance over-control gain. The entry re-targeting module computes the current trajectory and the capability footprint of the vehicle and determines if the selected landing site is within the capability of the vehicle. If the selected landing site falls outside of the capability of the vehicle, a new landing site is recommended to the crew. The entry display feeder completes the entry monitor modules in the guided entry domain and is the focus of this paper. The entry display feeder is the conduit between the GNC flight software and the onboard displays and operates at $10 \mathrm{~Hz}$ with all guidance modes, including manual control, while on the primary flight computer, and it will provide the minimum required functionality while on the backup flight computer.

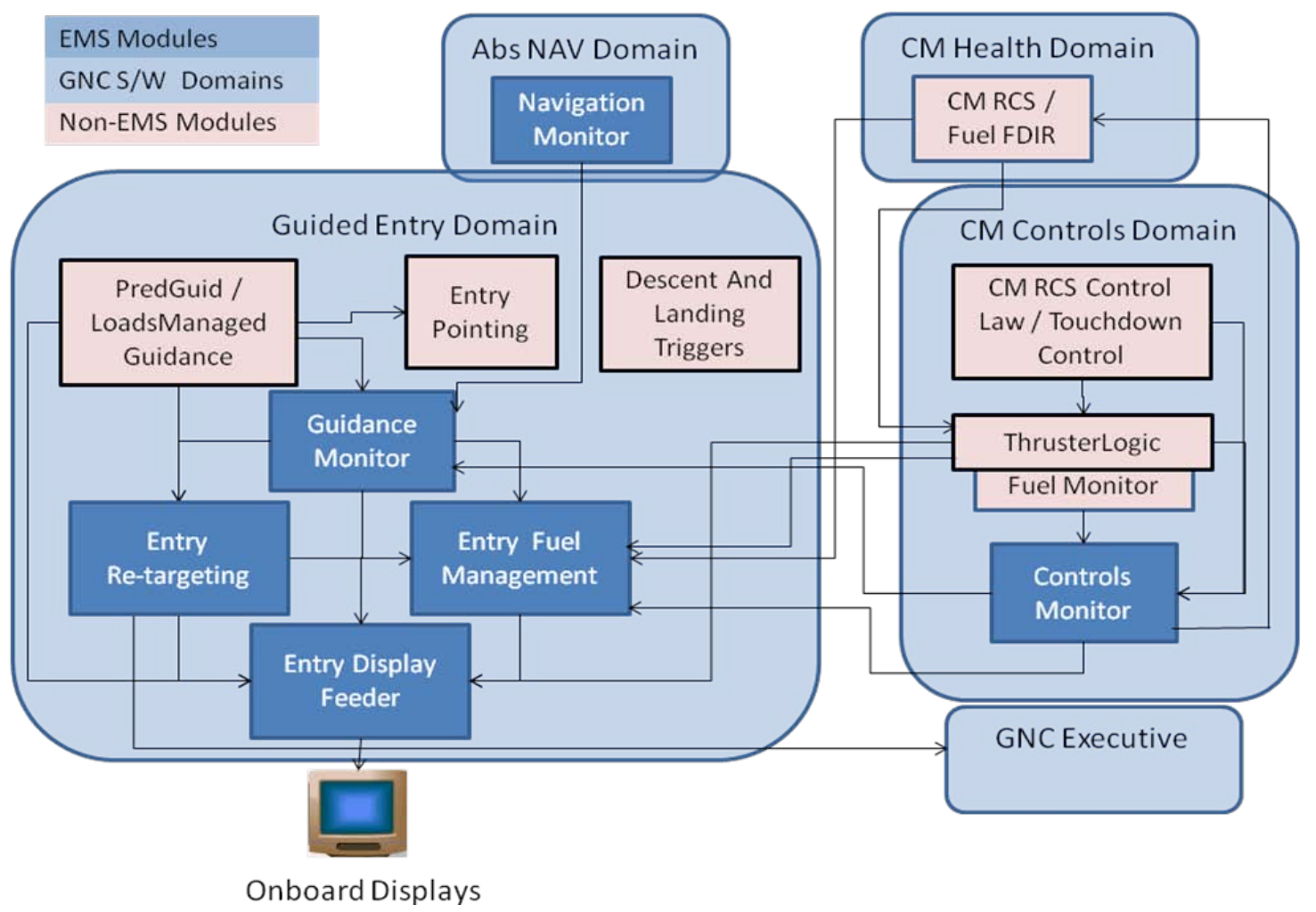

Figure 1 Flow Diagram of Conceptual Orion Entry Monitor System. 


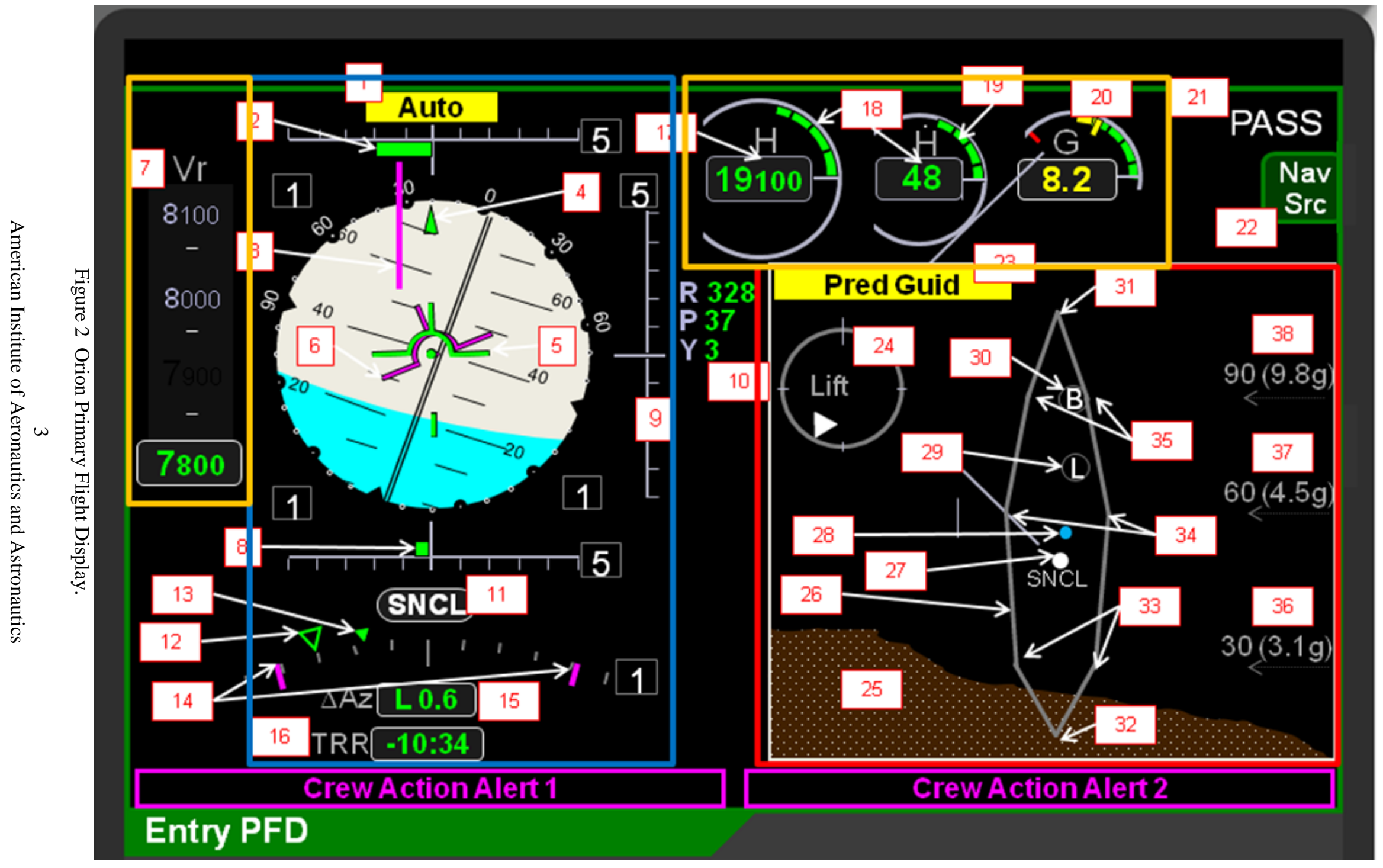


The landing is normally controlled autonomously, and the crew can fly precision entries manually in the event of an anomaly. The onboard entry displays will be used by the crew to monitor and, if necessary, manually fly the entry, descent, and landing. The entry trajectory displays, still under development, provide the crew with information about the energy of the trajectory, including an energy bucket and instantaneous impact points for a fulllift-up and full-lift-down trajectory. The PFD, the focus of this paper, is shown in Fig. 2, and the corresponding legend is shown in Table 1 [1]. The PFD provides the crew with information about the spacecraft attitude (blue box in Fig. 2), the entry trajectory and capability footprint (red box in Fig. 2), and additional flight control indicators (yellow boxes in Fig. 2).

\begin{tabular}{||l|l||}
\hline \multicolumn{2}{|c||}{ Table 1 Legend of PFD labels corresponding to Fig. 2.} \\
\hline 1 & \multicolumn{1}{|c||}{ Description } \\
2 & Automatic (AUTO) or manual (MANUAL) guidance \\
3 & Bank error scale \\
4 & Bank direction needle (smoothed) \\
5 & Current bank angle \\
6 & Reference attitude (fixed) \\
7 & Bank direction (unsmoothed) \\
8 & Relative velocity tape (green digits inside box indicates current) \\
9 & Yaw error scale \\
10 & Pitch error scale \\
11 & Digital readout of roll, pitch, yaw \\
12 & Selected landing site designator \\
13 & Current lateral angle \\
14 & Predicted lateral angle 10 seconds in future \\
15 & Lateral angle limits \\
16 & Digital readout of lateral angle from PredGuid \\
17 & Predicted time to roll reversal \\
18 & Digital readout of geodetic altitude \\
19 & Graphical and digital readout of geodetic altitude rate \\
20 & Graphical scale of geodetic altitude acceleration \\
21 & Axial acceleration with yellow and red g-load limit ticks (10 g's, 12 g's) \\
22 & Primary (PASS) or backup (BFS) flight software \\
23 & Navigation source block \\
24 & Guidance major mode \\
25 & Direction of lift vector (bank angle) \\
26 & Physical landmass of earth \\
27 & Vehicle landing footprint \\
28 & Location of selected landing site relative to landing footprint and earth mass \\
29 & Location of landing point as predicted by PredGuid entry \\
30 & Location of landing point as predicted by Loads Managed Guidance entry \\
31 & Location of landing point as predicted by Ballistic entry \\
32 & Location of landing point with full lift down trajectory \\
33 & Location of landing point with full lift up trajectory \\
34 & Location on landing footprint with \pm 30 degrees bank angle \\
35 & Location on landing footprint with \pm 60 degrees bank angle \\
36 & Location on landing footprint with \pm 90 degrees bank angle \\
37 & Peak acceleration sensed by vehicle with \pm 30 degrees bank angle \\
38 & Peak acceleration sensed by vehicle with \pm 60 degrees bank angle \\
\hline
\end{tabular}




\section{Attitude Indicators}

The spacecraft attitude indicators, attitude errors, and bank angle queues are shown in the blue outline. The Attitude Director Indicator (ADI) ball provides a 3D representation of the spacecraft attitude in a spacecraft reference frame. The digital readouts to the right of the ADI ball show the roll, pitch, and yaw attitudes of the vehicle. These attitudes are provided to the display as angles of bank, sideslip, and pitch from the absolute navigation domain. The corresponding attitude rates and errors are shown on the graduated bar scale on the top, right, and bottom of the ADI ball. During entry, the crew can manually control the bank angle but not the pitch and yaw angles. Pitch and yaw forces are dominated by aerodynamics effects and cannot be significantly affected by thrusters during entry, so those channels are disabled, and no rate error needles are shown for pitch and yaw.

\section{A. Bank Error Smoothing}

The green airplane is a fixed reference bank angle, and the magenta airplane represents the bank direction to remove the bank error. Bank error is computed within the entry display feeder as the difference between the actual and commanded bank angles. The vertical, magenta bank error needle also provides more precise bank error information, and the entry display feeder provides an option to smooth the bank error needle signal with a low-pass filter with a time constant of $2 \mathrm{~s}$ to prevent jumpiness that would distract a crew. Figure 3 shows the effect of varying the time constants of the filter. Smoothing with a time constant of $1 \mathrm{~s}$ is mostly acceptable but does not completely remove the jittering at all times, while a time constant of 3 s or greater results in signal attenuation and lag that analysis determined to cause undesirable errors in landing accuracy. Therefore, a time constant of $2 \mathrm{~s}$ is chosen as a reasonable compromise and is consistent with a recommendation provided by NASA Ames Research Center [2].

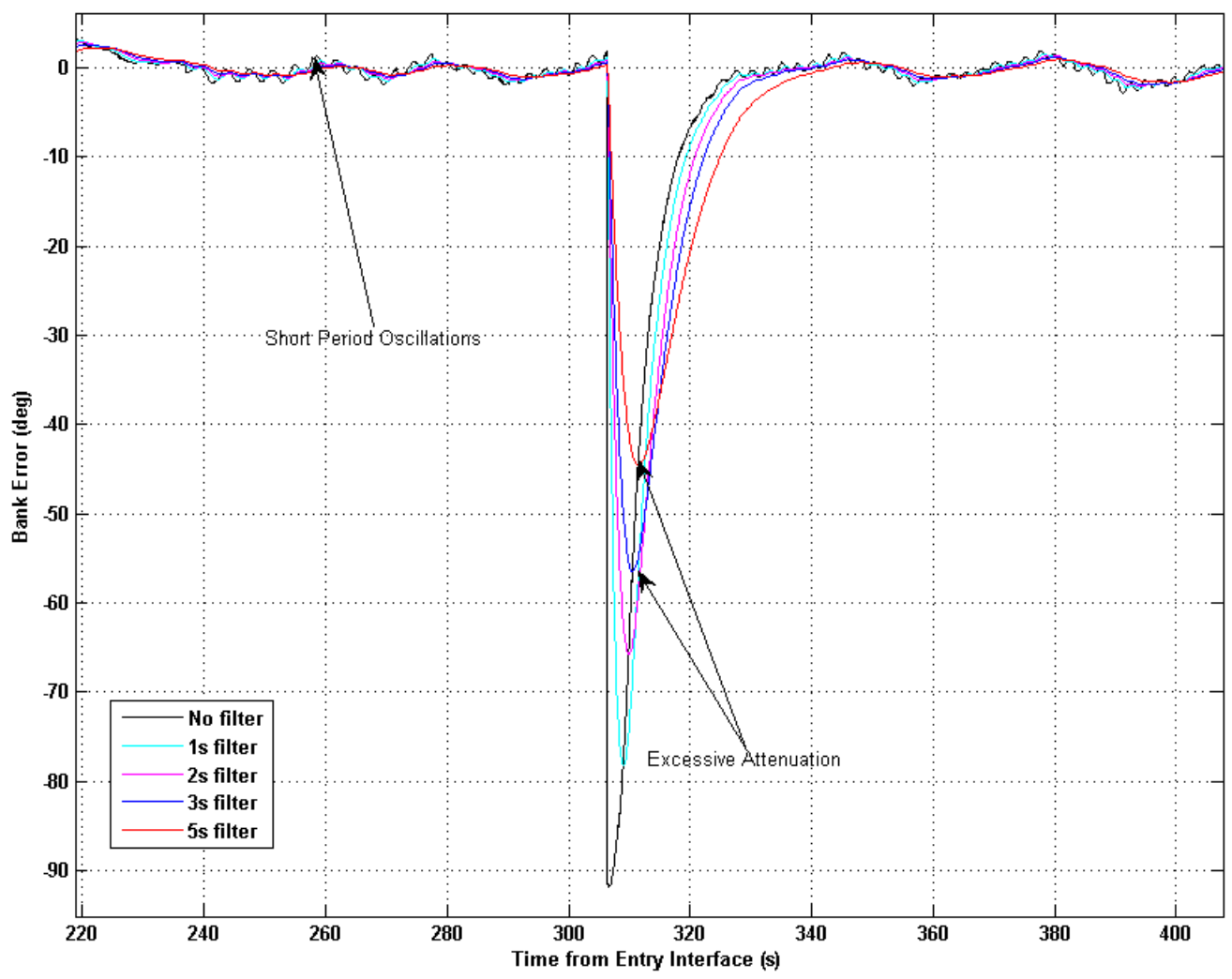

Figure 3 Effects of time constants on smoothing of bank error. 


\section{B. Bank Error Needle and Shortest Time Logic}

The vertical, magenta needle shows the bank angle error, which may be smoothed or unsmoothed, as described in the previous section. The entry display feeder also calculates the direction to bank consistent with the shortest time and least fuel consumption, which is not necessarily the direction of shortest angular distance, while operating independently of any guidance and control algorithms. This logic is useful during manual control and is consistent with the algorithm used by the flight control system $[3,4,5]$. The problem can be introduced as a time optimal bangbang control problem. Given the bank, bank rate, and constant roll acceleration, the switching line can be given as:

$u= \begin{cases}+1 & x-\frac{\dot{x}|x|}{2 a}>0 \\ -1 & x-\frac{\dot{x}|x|}{2 a}<0\end{cases}$

where $x=$ bank error and $a=$ constant acceleration magnitude

The algorithm includes phase wrapping where minimum time to bank left and bank right is calculated. Then $u$ is chosen based on shortest time. Figure 4 shows the state space trajectories and switching curves with phase wrapping.

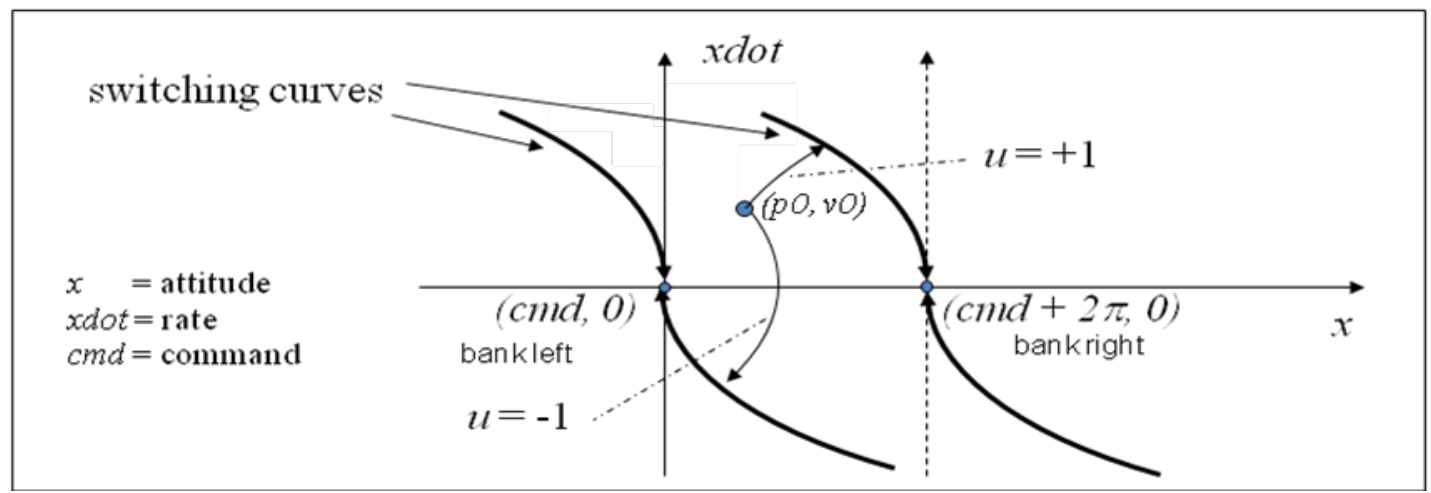

Figure 4 Description of switching curves.

However the problem is constrained by a vehicle rate limit. A peak rate is found when the time to bank is resolved. If the peak rate exceeds the rate limit, the time to bank is adjusted with a rate limited time to bank. Solutions for the left and right roll directions are compared until finally the appropriate bank error is selected. Figure 5 shows the flow of the shortest time logic as it is used in the display feeder. 


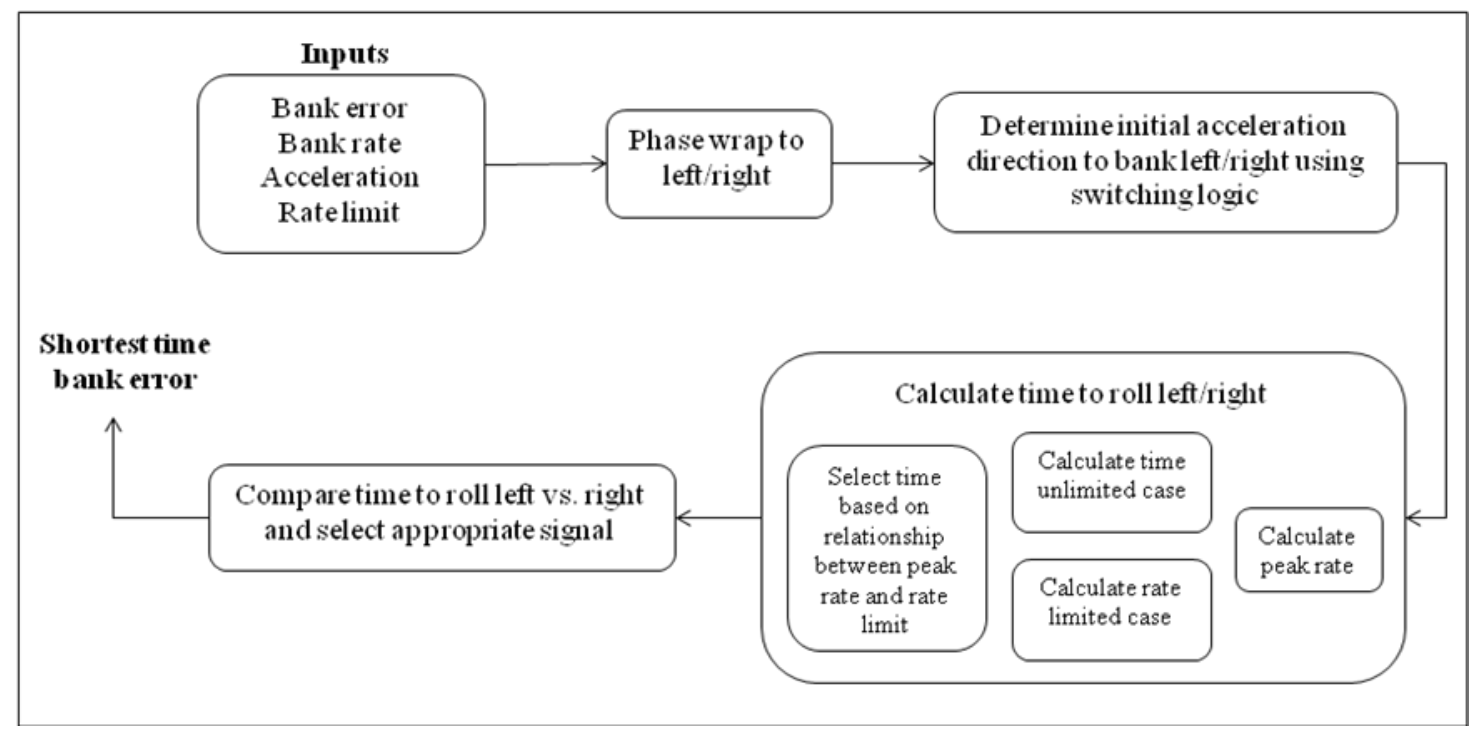

Figure 5 Flow diagram of shortest time logic for bank.

Figure 6 shows an example of the shortest time logic taking effect over the shortest distance solution. At 2166 seconds, the difference between the commanded bank and actual bank becomes just over 180 degrees as shown by the pre shortest time logic dashed line. However, after the shortest time logic, it can be seen that rolling right instead of left will yield the shortest time as shown by the blue solid line. The same occurs at 2232 seconds but for most of the profile shown, it can be seen that the shortest distance and shortest time solutions are the same. In this example, the shortest time logic solution produces a different solution only when the error is just over 180 degrees.
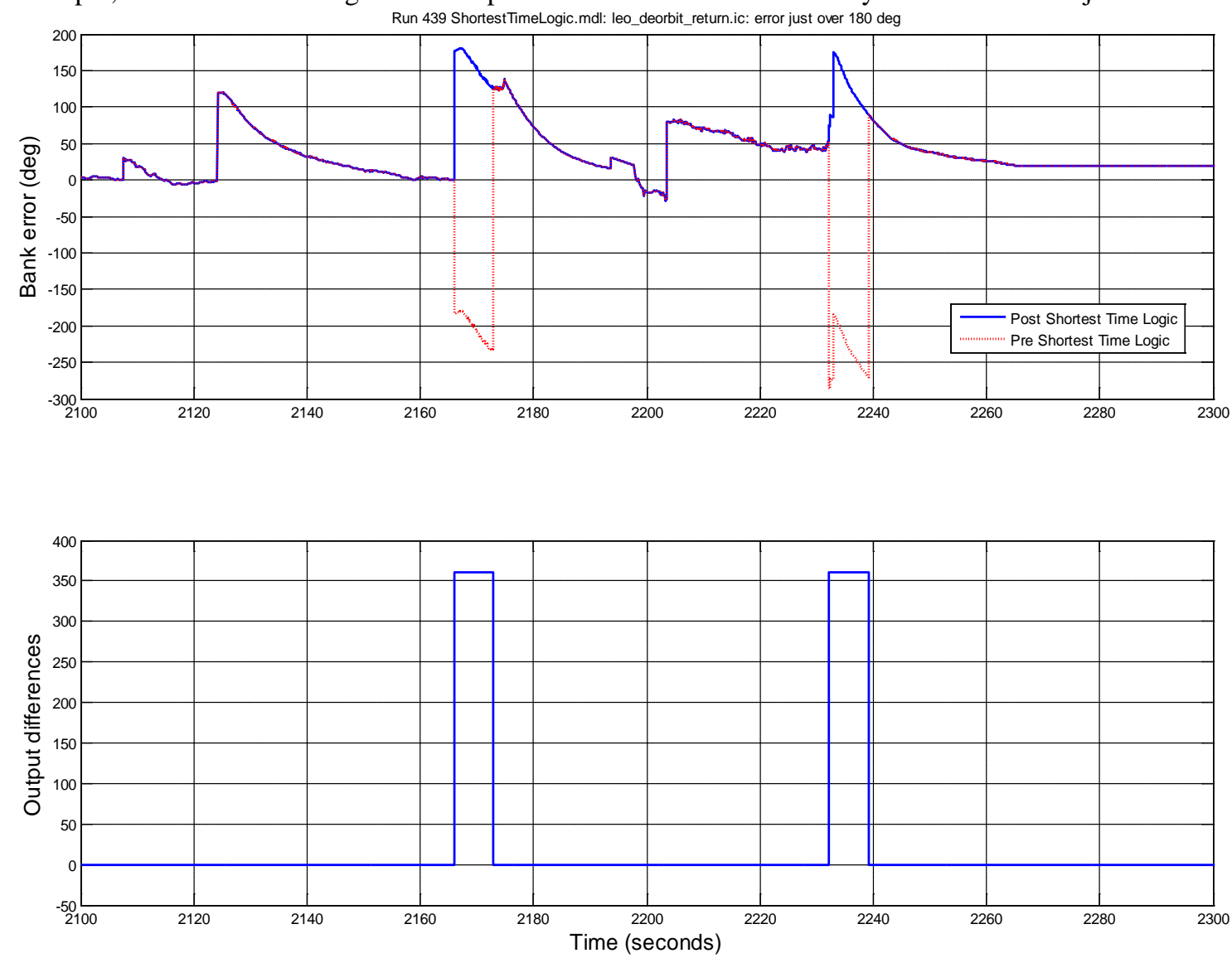

Figure 6 Example of shortest time logic taking effect.

American Institute of Aeronautics and Astronautics 


\section{Lateral Angle Prediction}

The heading error is computed as a lateral angle, which is the angle between the trajectory plane, determined by the vehicle position and velocity vectors, and the target plane, determined by the vehicle and target landing site position vectors [6]. When on the primary guidance algorithm, PredGuid, a bank reversal is triggered when the lateral angle exceeds the lateral corridor limits. The current lateral angle and lateral angle 15 seconds in the future are shown with respect to the lateral angle limits in the bottom left of the PFD, and these angles provide indications to the crew of when a bank reversal is likely to occur. The entry display feeder calculates the lateral angle in the future by propagating the current lateral angle rate, where the future time can be set with a parameter. The lateral angle and lateral angle prediction error for 15 seconds in the future is shown in Fig. 7a and Fig. 7b, respectively. Because the lateral angle may changes direction less than $15 \mathrm{~s}$ in the future, errors can grow to be as large as $0.2^{\circ}$ near the time of a bank reversal, and in this region the crew will use the time to bank reversal readout to better anticipate an upcoming bank reversal.
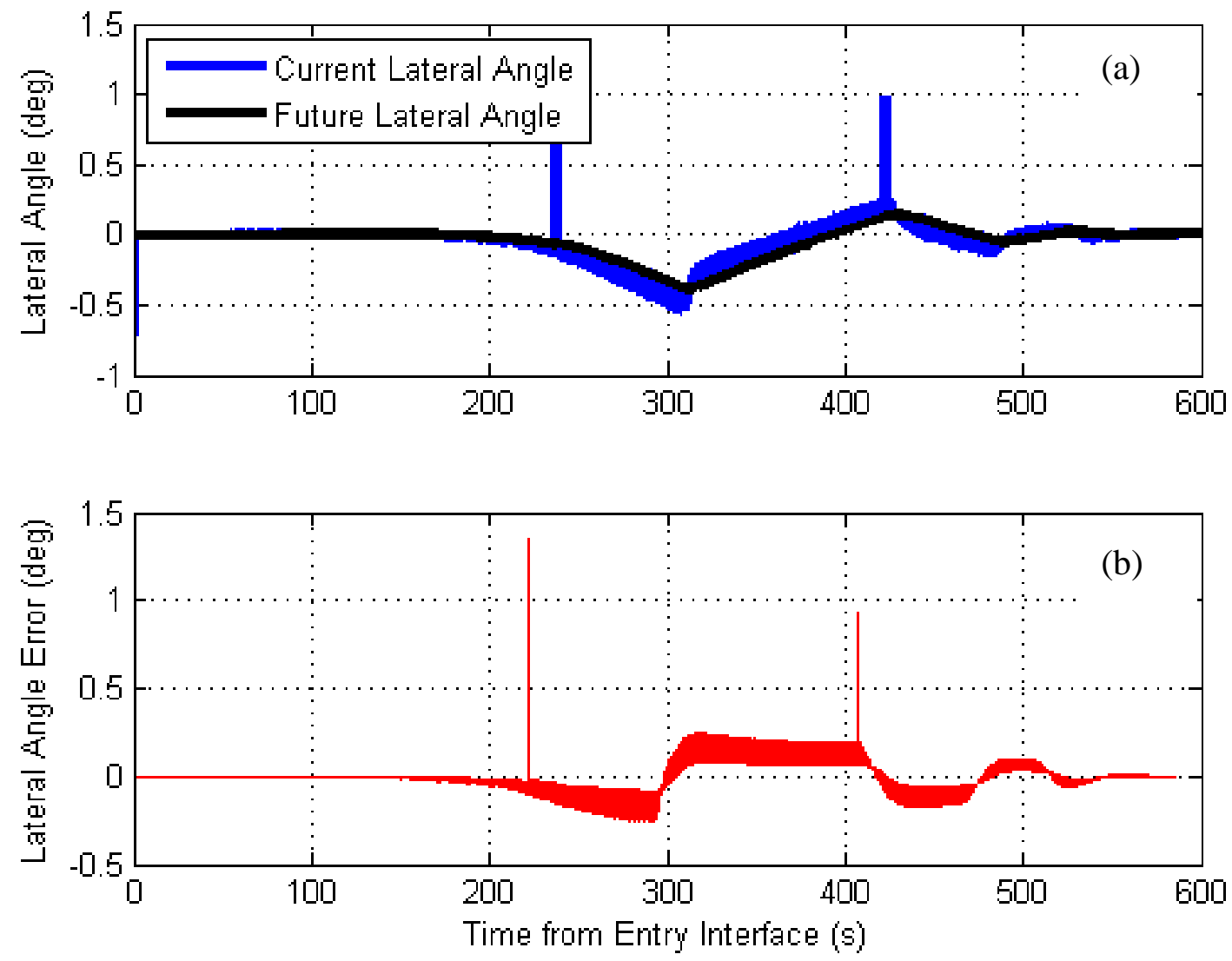

Figure 7 Lateral angle and errors.

\section{PredGuid Time to Bank Reversal Estimation}

To fly a precision entry the crew must have a means to anticipate the time to the next bank reversal, which is shown along with a digital readout of the current delta-azimuth on the digital display below the ADI ball. This estimate is based on the current lateral angle, current lateral angle rate, and lateral angle deadband. Figure 8 shows the lateral angle and lateral corridor throughout a nominal trajectory while on primary guidance (PredGuid). A bank reversal is triggered when the lateral angle exceeds the lateral angle deadband and is consistent with the primary entry guidance algorithm, PredGuid. To predict this time, the current lateral angle is propagated using the current lateral angle rate, and the time to reach the current lateral corridor is calculated assuming using current constant lateral angle rate. 


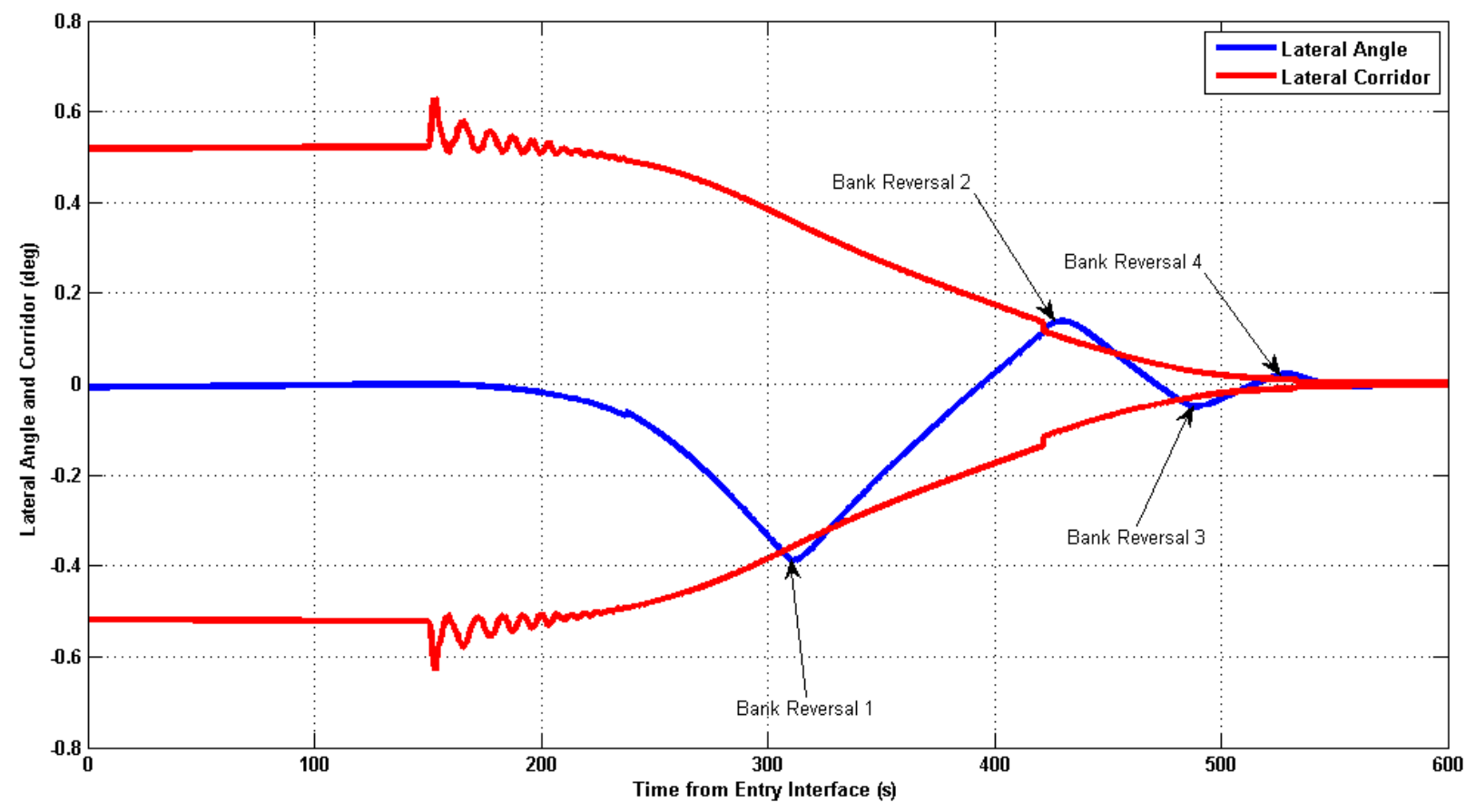

Figure 8 Lateral angle and lateral corridor on nominal PredGuid trajectory.

Figure 9 shows the estimation of the time to bank reversal for the nominal PredGuid ISS entry. When the lateral angle is outside of the lateral corridor the time to bank reversal remains " 0 ," which indicates to the crew that a bank reversal is or should be in progress. Figure 10 shows the errors in the estimation of the time to the first bank reversal, which are due to changing bank angles and the non-linear changes in the lateral corridors. The bank reversal occurs at EI $+306 \mathrm{~s}$. The crew is most interested in this indicator starting $10 \mathrm{~s}$ prior to the bank reversal, and the errors are a maximum of about $2.5 \mathrm{~s}$ at the start of this time period, reducing to about $0.25 \mathrm{~s}$ at the time of the bank reversal. An attempt was made to simultaneously solve the lateral angle prediction and lateral corridor prediction equations for time, based on current signal rates. This implementation resulted in higher errors due to the nonlinearity of the lateral corridor. These attitude indicators provide the crew with the visual queues to modulate bank in a manner consistent with PredGuid while flying manually and are of sufficient accuracy to provide the crew with situational awareness and an early warning of when to expect a bank reversal. 


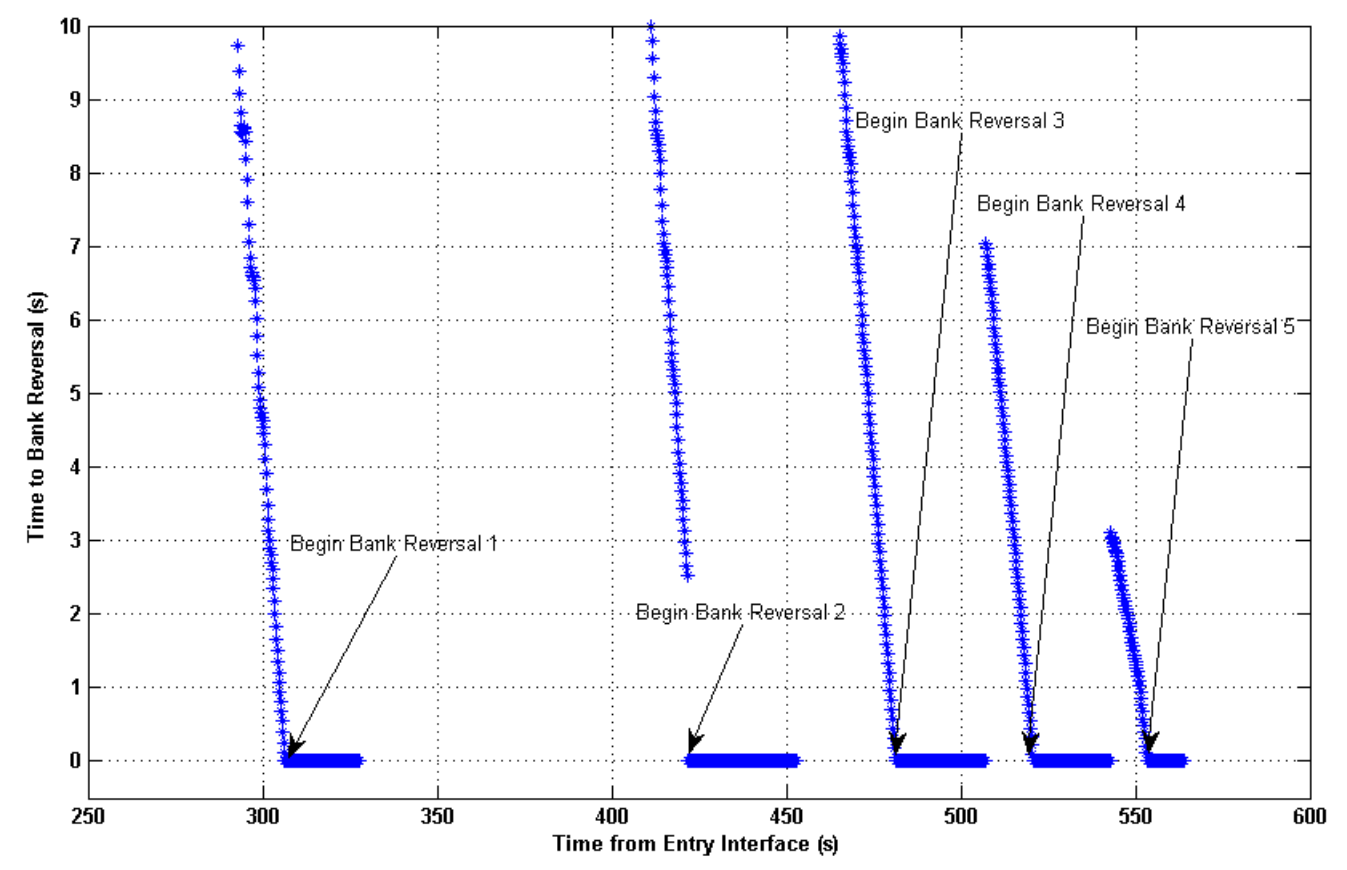

Figure 9 PredGuid Time to Bank Reversal

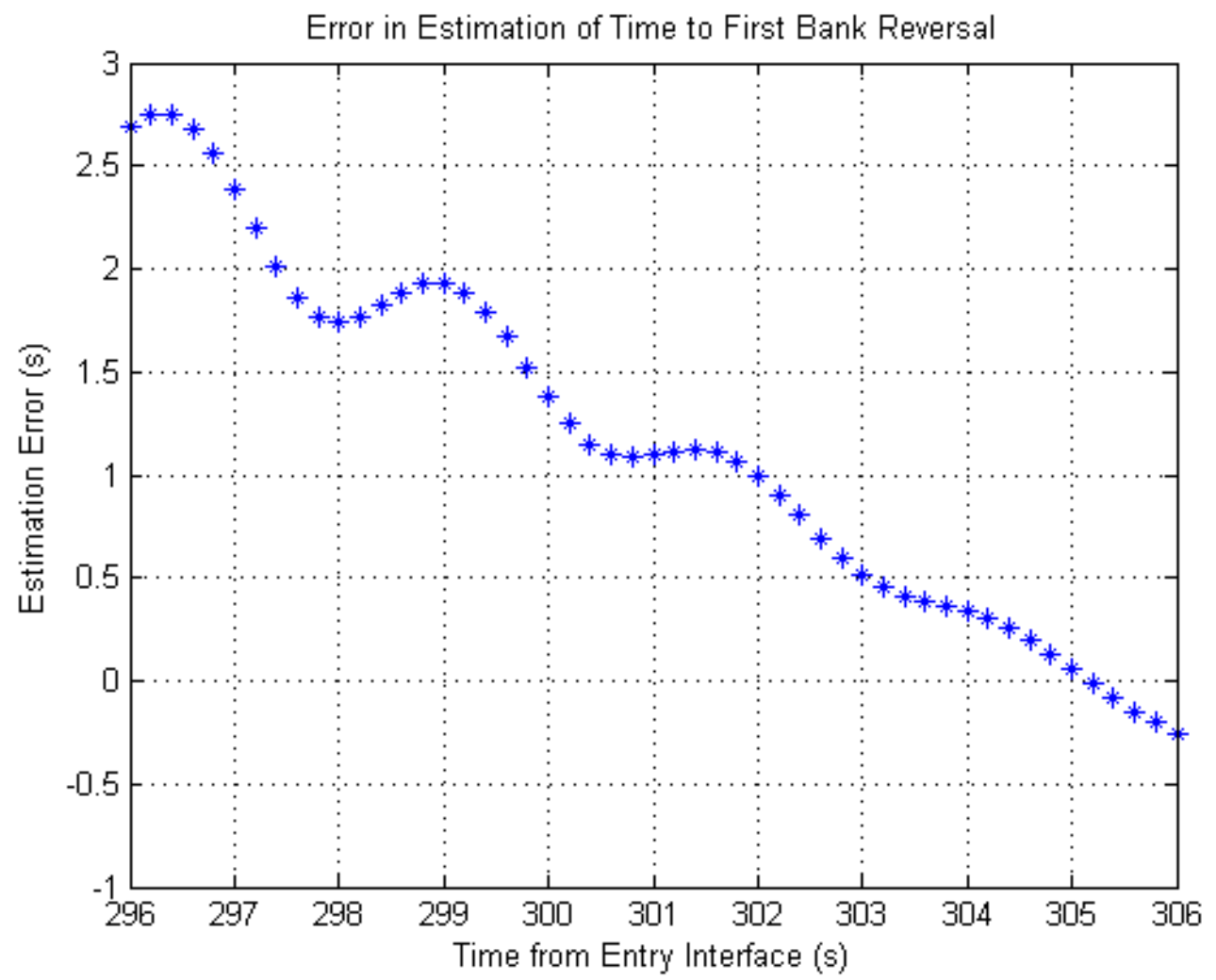

Figure 10 Errors in estimation of time to first bank reversal. 


\section{E. Precision Loads-Managed Guidance (PLMG) Time to Bank Reversal Estimation}

The backup guidance algorithm, Precision Loads Managed Guidance (PLMG) executes a single bank reversal at a relative velocity of $5700 \mathrm{~m} / \mathrm{s}$ to improve landing accuracy while still ensuring that load limits will not be violated at any point in the trajectory. The logic to calculate the time to bank reversal simplifies to predicting the time that the relative velocity reaches $5700 \mathrm{~m} / \mathrm{s}$ and does not rely on estimating a time at which a lateral corridor boundary will be crossed. Equation (1) is a variation of calculating time based on distance and velocity and describes the logic to calculate the time to the bank reversal while on PLMG, where Gload is the current axial acceleration of the vehicle in Gs, $p v 2$ is a coefficient from a least squares fit on the velocity profile, $V_{\text {rel_LMG }}$ is the relative spacecraft velocity, and $V_{\text {Bank }}$ is the defined relative velocity at which the bank reversal is triggered [7].

$$
\text { Time_To_Bank_Reversal_PLMG }=(\text { Gload } * p v 2) /\left(V_{\text {rel_LMG }}-V_{\text {Bank }}\right)
$$

Figure 11 shows the estimation of the time to bank reversal as a function of relative velocity and time from entry interface for the nominal PLMG ISS entry. The errors in this estimation are small and provide the crew with excellent situational awareness and advanced warning of when to expect a bank reversal. At 9 seconds prior to the bank reversal the time to bank reversal is estimated to be 9 seconds, and at 13 seconds prior to the bank reversal the time to bank reversal is estimated to be 14 seconds.

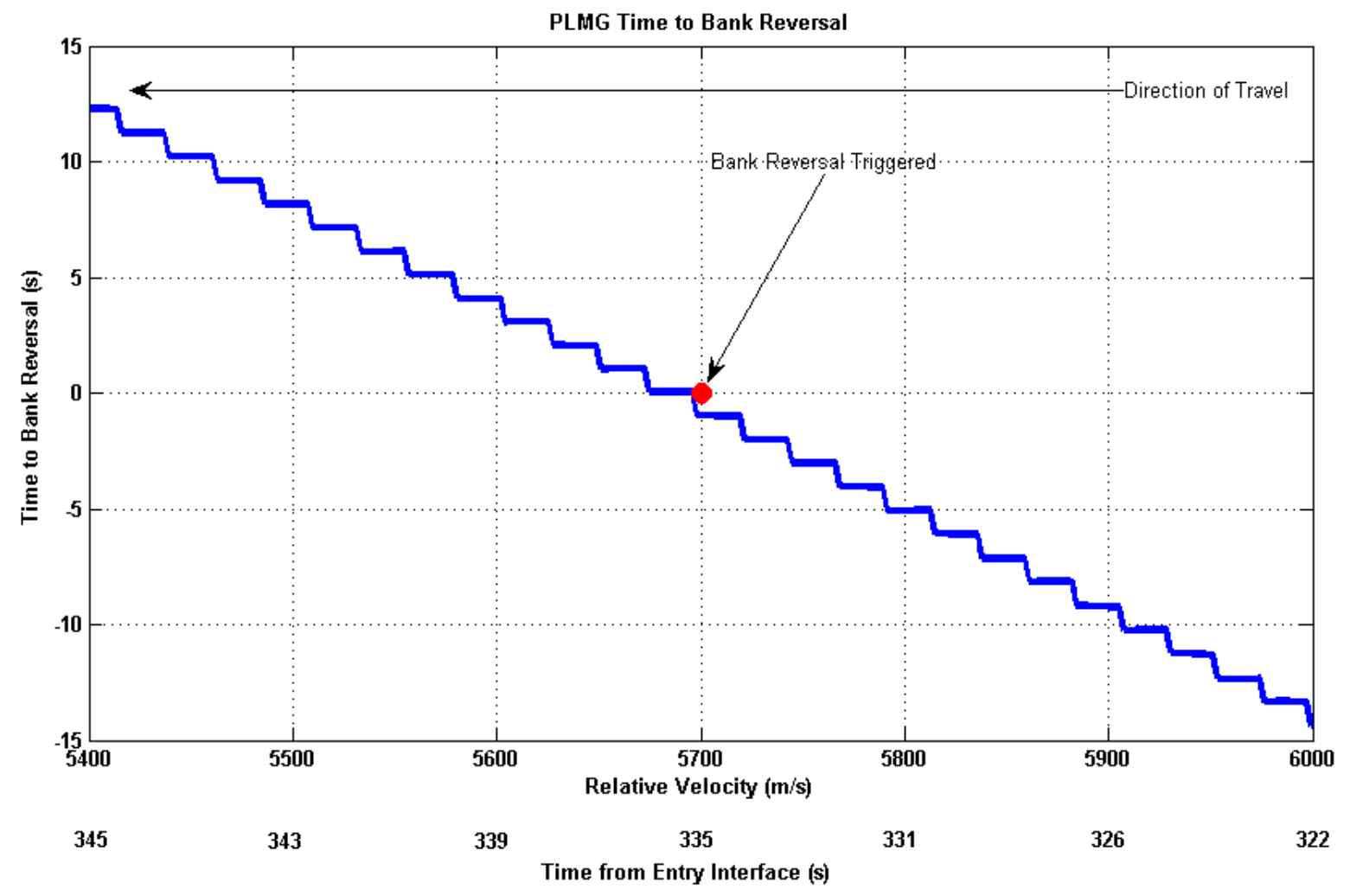

Figure 11 Time to Bank Reversal on PLMG.

\section{Entry Footprint Display}

The second area of the PFD consists of the entry footprint and bank angle displays, shown in the red outline on Fig. 1. The current lift direction, consistent with the current bank angle, is shown with an arrow on the dial display. The entry footprint display provides the crew with geographic situational awareness during entry. Because the crew is flying backwards through the atmosphere, consistency between the display orientation and bank angle directions is required. Therefore, the footprint is oriented upside down such that the California coastline will be shown on the bottom of the display. Throughout entry, the entry re-targeting module calculates all of the onboard trajectory 


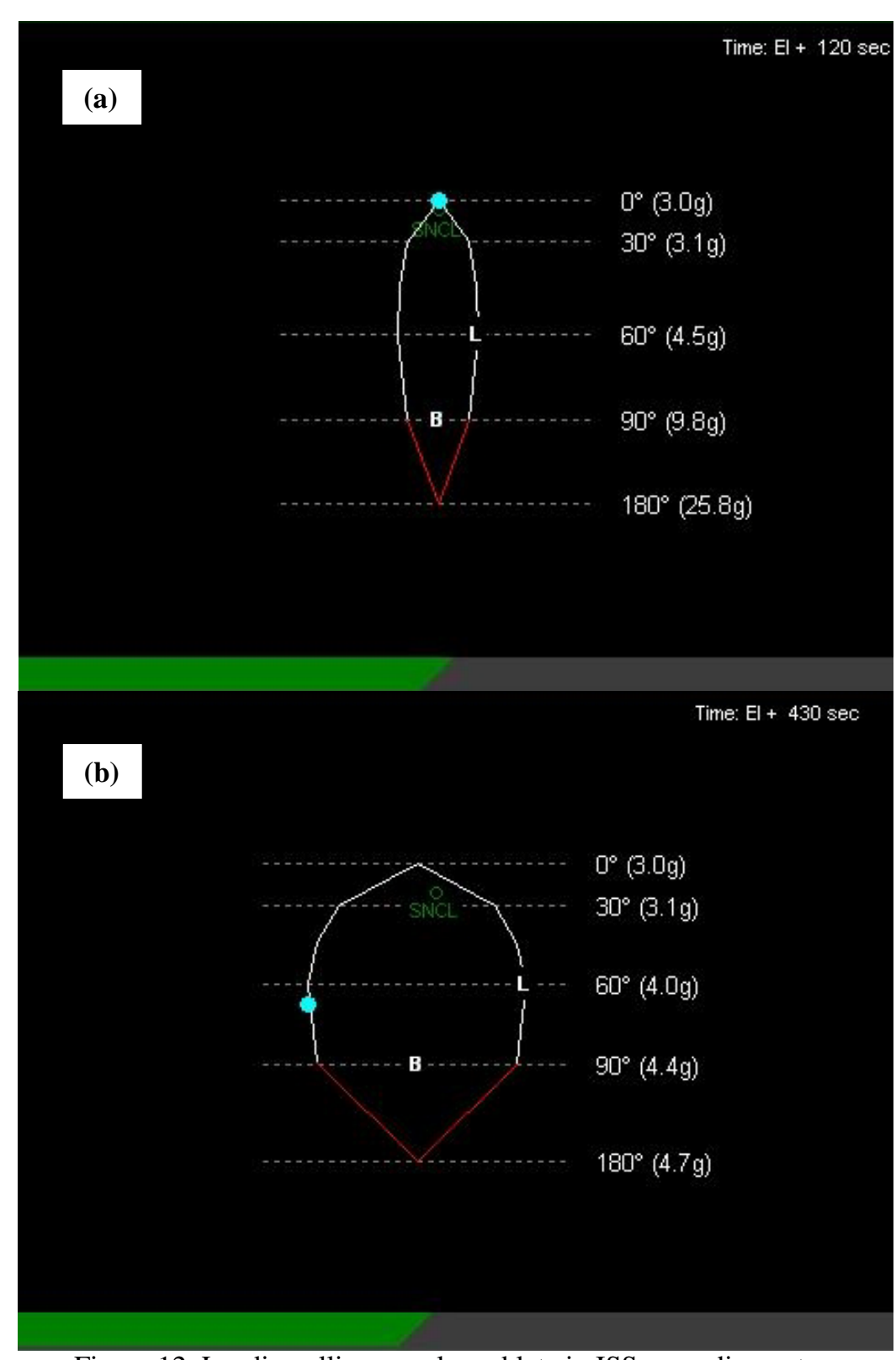

Figure 12 Landing ellipses early and late in ISS ascending entry. propagations and passes the data to the footprint display via the entry display feeder. The target landing site is indicated by the circle labeled "SNCL" (San Clemente Island), and the predicted landing site assuming a current, constant bank angle is indicated by the cyan circle. The predicted landing site on PLMG is indicated by the "L," and the ballistic landing site is indicated by the "B." to increase performance of the entry retargeting module, the ballistic landing point is not calculated by the entry re-targeting module. Instead, the ballistic landing site is calculated within the entry display feeder as the midpoint of the great circle arc defined by the $+90^{\circ}$ and $90^{\circ}$ landing points. The landing capability footprint is indicated by the closed polygon, which shows the predicted landing sites at constant bank angles of $0^{\circ}$ (full lift up), $\pm 30^{\circ}, \pm 60^{\circ}, \pm 90^{\circ}$, and $180^{\circ}$ (full lift down). To the right of the footprint are the constant bank angle indicators which would result in the capsule landing at that point on the ellipse. The corresponding maximum G-loads that would be encountered when flying these constant-bank trajectories to parachute deploy are shown to the right of the footprint and are calculated by the entry retargeting module. Figures 12a and $12 \mathrm{~b}$ show the landing ellipse at 120 seconds and 430 seconds after entry interface, respectively. As energy is removed, the landing

capability of the vehicle shrinks dramatically. The footprint is scaled on the display to maintain a constant length (from toe to heel) at all times.

When the target landing site falls outside of the capability footprint due to a spacecraft fault or trajectory error, the onboard re-targeting module of the EMS recommends a new landing site to the crew via an alert in the yellow area of the footprint display. It recommends the safest reachable re-targeted landing site based on a pre-loaded list of contingency landing sites. If none of those sites are available, the re-targeted landing site is recommended based on a relative factor of safety matrix that underlies the entry footprint display. The footprint display provides the crew with the visual queues to manually fly to the safest possible landing site within the capability of the vehicle.. Looking at the indicators to the right of the footprint display, the crew can control latitude and maximum G load. These queues are important to the crew because the PredGuid algorithm is currently not designed to accept a new landing site target after entry interface. For ISS entries, the yellow G-load limit will likely not be violated if the bank angle is held at or below $90^{\circ}$, and the crew will have the option of exceeding this yellow limit at their discretion. 


\section{Additional Flight Control Indicators}

The third area of the PFD consists of basic flight control indicators, excluding attitude indicators. Most of these data are passed directly from the absolute navigation domain of the GNC flight software to the displays via the entry display feeder and are shown in the orange outlines on Figure 1 . The only exception is the altitude acceleration, which is computed within the display feeder by differentiating the altitude rate.

In the center of the dial displays, digital readouts of the altitude, altitude rate, and current acceleration (G-load) are shown. Altitude rate is also shown in the ticks of the altitude dial, and altitude acceleration is shown in the ticks of on the altitude rate dial. The yellow and red G-load limits of $10 \mathrm{Gs}$ and $12 \mathrm{Gs}$, respectively, are shown on the acceleration dial indicator. Earth-relative velocity in $\mathrm{ft} / \mathrm{s}$ is shown on a tape display to the left of the ADI ball, where the current relative velocity is shown in the highlighted box at the bottom of the tape. These basic flight control indicators complement the other displays on the PFD and provide the pilots with additional situational awareness necessary to fly an entry manually.

\section{Conclusion}

The entry display feeder is an integral part of the Orion EMS and serves as a conduit between multiple GNC flight software domains and the displays. It is responsible for outputting data in the proper units and also generates display data for the PFD that are not provided by any other flight software modules. Notable features include smoothing of the bank error data, shortest time logic in the bank error signal to improve fuel efficiency and performance when manually banking during entry, prediction of the time to bank reversal under both PredGuid and PLMG, and prediction of the lateral angle $15 \mathrm{~s}$ in the future.

The entry display feeder is being extended to feed the entry trajectory displays, which provide additional insight into the energy state of the vehicle during entry. Further enhancements to the entry display feeder are under consideration and include smoothing of the lateral corridor to reduce oscillations in the time to bank reversal predictions. Improvements to the performance of the entry retargeting module will also be investigated to increase the processing speed of the landing footprint data, and an extra propagation may be added to display the landing location using a $180^{\circ}$ bank angle and a constraint of $10 \mathrm{Gs}$ throughout the entry profile.

\section{Acknowledgments}

The authors would like to thank Zach Putnam of Draper Labs and Mike Tigges of NASA Johnson Space Center for their advice on improving the accuracy of the time to bank reversal and future lateral angle predictions while on PredGuid. The authors would also like to thank Wyatt Johnson of NASA Johnson Space Center for his advice on implementing the logic to determine the time to the bank reversal while on PLMG. The authors thank Tim Verborgh for building the displays and working tirelessly with the EMS team to troubleshoot the display feeder and ANTARES simulation in support of the 2010 Orion handling qualities assessments. The authors also thank Karl Bilimoria of NASA Ames Research Center for providing advice on implementing the bank error smoother.

\section{References}

${ }^{1}$ Matthews, D., “Ascent Abort Flight Display Suite, Pass 1 Description,” NASA Johnson Space Center, 2009.

${ }^{2}$ Bilimoria, K., email, 11 September 2009, karl.bilimoria@nasa.gov.

${ }^{3}$ Bryson, A E, Jr. and Ho, Y C. Applied Optimal Control: Optimization, Estimation, and Control, Hemisphere, 1975, pp. 111113.

${ }^{4}$ Loe, G., e-mail, 16 March 2010 and 26 March 2010, Greg.Loe@honeywell.com.

${ }^{5}$ Yazell, D., e-mail, 04 May 2010 and 26 March 2010, Douglas.Yazell@honeywell.com.

${ }^{6}$ Putnam, Z.R., Neave, M.D., and Barton, G.H., "PredGuid Entry Guidance for Orion Return from Low Earth Orbit," IEEE paper \#1571, 7 January 2009.

${ }^{7}$ Johnson, W., Personal Interview, 5 April 2010. 